\title{
Trading Strategies within the Edges of No-Arbitrage
}

\author{
Álvaro Cartea ${ }^{\mathrm{a}}$, Sebastian Jaimungal ${ }^{\mathrm{b}}$, Jason Ricci ${ }^{\mathrm{c}}$ \\ ${ }^{a}$ Mathematical Institute, University of Oxford, Oxford OX2 6GG, UK \\ ${ }^{b}$ Department of Statistics, University of Toronto, Toronto M5T 1P5, Canada \\ ${ }^{c}$ Department of Statistics, University of Toronto, Toronto M5T 1P5, Canada
}

\begin{abstract}
We develop a trading strategy that employs limit and market orders in a multi-asset economy where the assets are not only correlated, but can also be structurally dependent. To model the structural dependence, the mid-price processes follow a multivariate reflected Brownian motion on the closure of a no-arbitrage region which is dictated by the bid-ask spreads of the assets. We provide a mathematical framework for such an economy and solve for the value function and optimal control for an investor who takes positions in these assets. The optimal strategy exhibits two dominant features which depend on how far the vector of mid-prices is from the no-arbitrage bounds. When mid-prices are sufficiently far from the no-arbitrage edges, the strategy behaves as that of a market maker who posts buy and sell limit orders. And when the mid-price vector is close to the edge of the no-arbitrage region, the strategy executes a combination of market orders and limit orders to profit from statistical arbitrages. We discuss a numerical scheme to solve for the value function and optimal control, and perform a simulation study to discuss the main characteristics of the optimal strategy.
\end{abstract}

Keywords: Optimal trading; High-frequency trading; Algorithmic trading; Limit orders; Market orders; Stochastic control; Impulse control; No-arbitrage bounds.

\footnotetext{
This work expresses the opinion of its authors and is in no way representing the opinion of the institutions the authors work for. ÁC acknowledges the research support of the Oxford-Man Institute for Quantitative Finance and the hospitality of the Finance Group at Saïd Business School. SJ would like to thank NSERC and GRI for partially funding this work. The authors would like to Robert Almgren for comments on an earlier version of this article. We also thank conference and seminar participants at University College London, University of Toronto.

Email addresses: Alvaro.Cartea@maths.ox.ac.uk (Álvaro Cartea), sebastian.jaimungal@utoronto.ca (Sebastian Jaimungal), jason.ricci@mail.utoronto.ca (Jason Ricci)
} 


\section{Introduction}

Over the past decade, advances in technology and computing power have given rise to a new age in trading. There has been a movement from traditional pit exchanges to electronic exchanges where open quotes get amalgamated into a limit order book (LOB) and agents trade by placing limit orders (LOs) with intent to buy/sell at a particular price or by sending market orders (MOs). In this paper, we develop a strategy where the investor trades in a collection of assets which are not only correlated, but present static arbitrage opportunities if their mid-prices stray outside a no-arbitrage region which is dictated by the bid-ask spreads of the assets.

The investor's goal is to maximize cash profits at some terminal time $T$, while controlling for inventory risk by penalizing open positions. The investor may trade aggressively and take liquidity via MOs if she sees a profitable opportunity in the form of a statistical arbitrage, or may trade passively by submitting limit buy and sell orders to the LOB. The trading strategy requires aggressive or passive orders depending on where the mid-prices are relative to the boundaries of the no-arbitrage region. The following two examples illustrate how certain structural dependencies in a collection of assets determine the no-arbitrage region.

Suppose that an investor maximizes expected wealth by trading in an equity futures contract which matures at time $T_{1}$ and also trades in its underlying. Furthermore, assume that this underlying equity does not pay dividends and the risk-free rate is zero. Let $S_{t}^{1}$ denote the price of the underlying at time $t$ and $S_{t}^{2}$ the price of the futures contract. In an economy where assets are traded with a zero bid-ask spread (that is, the price to sell or buy the asset is the same), we have, by no-arbitrage, $S_{t}^{1}=S_{t}^{2}$ for all $t \leq T_{1}$. If this equality does not hold, then there exists an arbitrage.

Now assume that the underlying equity and its futures contract trade with bid-ask spreads $\xi_{1}>0$ and $\xi_{2}>0$, respectively. In this case, it is possible for the market to bear arbitrage-free prices of the form $S_{t}^{1} \neq S_{t}^{2}$. To see this, assume that $S_{t}^{1}>S_{t}^{2}$. To arbitrage these prices the investor sends a buy market order (MO) for the futures contract, for which she must cross the spread and pay $S_{t}^{2}+\xi_{2} / 2$, and simultaneously sends a sell MO for the underlying and obtains $S_{t}^{1}-\xi_{1} / 2$. This trade is an arbitrage if

$$
\left(S_{t}^{1}-\frac{\xi_{1}}{2}\right)-\left(S_{t}^{2}+\frac{\xi_{2}}{2}\right)>0 .
$$

Hence, it is simple to show that the no-arbitrage region is

$$
\left|S_{t}^{1}-S_{t}^{2}\right|<\frac{1}{2}\left(\xi_{1}+\xi_{2}\right)
$$

Another example is one in which an investor takes positions in the following three derivatives: 
i) The first nearby futures contract written on an equity having maturity $T_{1}$. ii) The second nearby futures contract written on the same equity having maturity $T_{2}>T_{1}$. iii) The 1-2 calendar spread contract having maturity $T_{1}$. The 1-2 calendar spread is a derivative that forces one party to exchange the $T_{2}$ maturity contract for the underlying asset at time $T_{1}$. Its payoff can be decomposed into being long the $T_{1}$ contract and short the $T_{2}$ contract. Furthermore, denote the mid-prices of these three contracts by $S_{t}^{1}, S_{t}^{2}, S_{t}^{3}$ and their spreads $\xi_{1}, \xi_{3}, \xi_{3}$, thus the no-arbitrage region is

$$
\left|S_{t}^{1}-S_{t}^{2}-S_{t}^{3}\right|<\frac{1}{2} \sum_{j=1}^{3} \xi_{j} .
$$

Below we provide a definition of the no-arbitrage region.

Midprices outside the no-arbitrage region are immediately corrected by arbitrageurs who exert price pressure until these opportunities disappear. In our framework, this arbitrage pressure is modelled by assuming that mid-prices follow a multivariate reflected Brownian motion on the closure of the no-arbitrage region. Thus, when the mid-price of an asset approaches the edges of the no-arbitrage region, market forces will exert pressure so that the mid-price is pulled back into the interior of the region.

We solve the investor's optimal investment strategy and show how it depends on the proximity of the mid-prices to the edges of the no-arbitrage region. When mid-prices are 'far' from the no-arbitrage boundary, the investor behaves as a strategic market maker who posts two-sided LOs. However, these LOs are strategically placed in the book to not only control for inventory exposure, but also in anticipation of mid-prices approaching the edges of the no-arbitrage region where the strategy employs a mix of market and LOs to execute statistical arbitrages. And when the mid-price of one asset is 'close' to the no-arbitrage boundary, the investor adjusts the depths of her LOs and may also execute MOs to take advantage of statistical arbitrage opportunities in anticipation of price pressures which force prices to stay within the no-arbitrage bounds, and to control exposure to inventory risk.

Our work is related to the literature that investigates investment strategies based on a collection of co-integrated assets (see for example Tourin and Yan (2013), Tourin and Lintilhac (2017), Cartea et al. (2015), Leung and Li (2015), and Cartea and Jaimungal (2016)), but our approach is different because we focus on a collection of assets with stronger structural dependencies, and more importantly, we model the no-arbitrage region and the investor employs LOs and MOs in the strategy. Other related literature in investment strategies specific to trading in a single asset to optimally liquidate/acquire shares as in Almgren and Chriss (2000), Almgren (2003), Kharroubi and Pham (2010), Guéant et al. (2012), Bayraktar and Ludkovski (2014), Cartea and Jaimungal (2015a), or in a general profit/utility maximization setting as in Avellaneda and Stoikov (Avellaneda and Stoikov), Guilbaud and Pham (2013), and Cartea et al. (2014). 
The rest of this paper is organized as follows. Section 2 contains definitions and some modeling assumptions. Section 3 states and discusses properties of the optimal trading strategy. Section 4 discusses the discretization scheme and convergence to the viscosity solution. Section 5 analyzes qualitative features in the interior and edges of the no-arbitrage in the two-dimensional case, and uses simulations to study the financial performance of the optimal strategy. Section 6 concludes and we collect some additional details of proofs in the Appendix.

\section{Trading in a basket of assets}

Here we develop a framework for investors who trade in a basket of assets. Our main focus is on assets that exhibit strong structural dependencies as in the examples above where the investor trades in futures contracts with the same underlying asset. The main question we answer is: How can an investor design an algorithmic strategy to take advantage of this structural dependency between assets?

We assume that the economy trades $n$ risky assets with mid-prices at time $t$ given by $S_{t}^{i}$, $i=1, \cdots, n$, and respective bid-ask spreads $\xi_{i}>0$, both of which we arrange in $n$-dimensional vectors $\mathbf{S}_{t}$ and $\boldsymbol{\xi}$ respectively. We also assume the existence of a risk-free cash asset that offers zero return and trades with zero bid-ask spread.

A well-known result in finance theory is that when one asset's payoff can be replicated as a linear combination of other traded assets, then there is a redundant asset in the economy. However, if some of these assets trade with a bid-ask spread, this redundancy ceases to exist. We classify a multiple asset economy as over-complete if there exists a non-zero portfolio and a finite investment horizon $\bar{T}$ such that at time $\bar{T}$ the portfolio's value is a constant, i.e. its variance is zero.

All random variables and stochastic processes in this paper are defined on the completed, filtered probability space $\left(\Omega, \mathcal{F}_{\bar{T}}, \mathbb{F}, \mathbb{P}\right)$ with filtration $\mathbb{F}=\left\{\mathcal{F}_{t}\right\}_{0 \leq t \leq T}$ and where $\mathbb{P}$ is the realworld probability measure. Note that the filtration $\mathbb{F}$ is only defined up to time $T<\bar{T}$, whereas $\mathbb{P}$ is defined on the larger $\sigma$-algebra $\mathcal{F}_{\bar{T}}$. Here, $\mathbb{F}$ is the natural filtration generated by $\left(\mathbf{S}_{t}, \mathbf{M}_{t}^{ \pm}, \mathbf{N}_{t}^{ \pm}\right)$, all of these processes will be defined later. As usual, equalities and inequalities among random variables are understood as holding on a $\mathbb{P}$-a.s. basis unless otherwise stated. In the sequel the operation $\cdot$ is the inner product on $\mathbb{R}^{n}$.

Definition 1. A financial system is said to be $\underline{\text { over-complete }}$ if $\exists \mathbf{b} \in \mathbb{R}^{n}, \bar{T}>0$ with $\|\mathbf{b}\|>0$ such that $\mathbb{V}\left[\mathbf{b} \cdot \mathbf{S}_{\bar{T}}\right]=0$, provided $\mathbf{S}_{\bar{T}} \in L^{2}\left(\Omega, \mathcal{F}_{\bar{T}}, \mathbb{P}\right)$, where $\mathbb{V}$ is the variance operator.

One of our motivating examples (first two nearby futures and the calendar spread) fits into 
this framework with portfolio $\mathbf{b}=(1,-1,-1)$ and $\bar{T}$ being the maturity of the first nearby futures. The illustrative example that will be used throughout this paper (first nearby futures and the underlying equity) also fits into this framework with $\mathbf{b}=(1,-1)$ and $\bar{T}$ being the maturity of the futures contract.

In Definition 1, the trivial portfolio $\mathbf{b}=\mathbf{0}$ is excluded from $\mathbb{R}^{n}$ since $\mathbb{V}\left[\mathbf{0} \cdot \mathbf{S}_{\bar{T}}\right]$ is zero, making every economy over-complete and rendering this classification trivial. Notice that if the economy does not admit such a portfolio (i.e. there does not exist such a vector $\mathbf{b}$ ), then this problem reduces to a (correlated) multiple asset problem, see for example Tourin and Yan (2013), and Cartea and Jaimungal (2016). The no-arbitrage region in this simplified case would be all of $\mathbb{R}^{n}$. Also observe that the set from Definition 1 consists of all the riskless portfolios when considering $\bar{T}$ as the investment horizon. However, $\bar{T}$ may be the only investment horizon where these portfolios are riskless.

Definition 2. The space $B$ is the set of all vectors $\mathbf{b}$ from Definition 1 plus the origin.

We require the set $B$ in Definition 2 to include the origin so that it is, in fact, a subspace. One technical point that is useful later on is that $B$ is a subspace of $\mathbb{R}^{n}$ and hence has an orthogonal basis and an orthogonal compliment.

In the simplified case where the economic system is not over-complete, the set $B$ would only consist of the origin. Note that the proposed model and strategies derived in this paper still apply in this simplified case without any modifications. The more interesting case is when $\operatorname{dim} B>0$ (i.e., the market is in fact over-complete) because this economy has an inherent structure that should not be overlooked. For this reason, our illustrative examples will pertain to economies that are over-complete.

An arbitrage refers to the ability to construct a portfolio with no initial cost, no chance of a loss, and a positive probability of gain. The precise definition is given as follows.

Definition 3. An arbitrage is a self-financing portfolio $\Pi$ that, for some $t \in[0, \bar{T}]$, satisfies the following conditions:

(i) $\Pi_{0}=0$,

(ii) $\mathbb{P}\left[\Pi_{t} \geq 0\right]=1$,

(iii) $\mathbb{P}\left[\Pi_{t}>0\right]>0$. 
As our economy also includes a cash asset that pays/receives zero interest and has zero bidoffer spread, this allows us to shift portfolios $\Pi$ by a constant, making it easier to satisfy condition (i) in Definition 3 after any spreads are paid at time 0 to initiate a position.

Definition 4. The no-arbitrage region, denoted by $\mathcal{A} \subset \mathbb{R}^{n}$, is the set of mid-prices that makes this economy arbitrage free.

It is worth pointing out that the no-arbitrage region is open. More specifically, $\mathcal{A}$ is the set of mid-prices such that the initial cashflow from purchasing a portfolio $\mathbf{b}$ plus the terminal payoff, $\mathbf{b} \cdot \mathbf{S}_{\bar{T}}$, is strictly negative, whenever this portfolio matures at a constant value (i.e. when $\left.\mathbb{V}\left[\mathbf{b} \cdot \mathbf{S}_{\bar{T}}\right]=0\right)$.

This observation deserves some explanation. It is clear that the no-arbitrage set $\mathcal{A}$, as previously described, is open. At the boundary, these portfolios have cost equal to their maturity value. Also, it has the potential for arbitrage profits via the following strategy: Obtain portfolio $\mathbf{b}$ now for cost $\mathbf{b} \cdot \mathbf{S}_{\bar{T}}$. There is a positive probability that prior to $\bar{T}, \mathbf{S}_{t}$ will move to a value (within $\mathcal{A}$ ) such that a filled limit order (LO) on one asset, combined with liquidation of the others assets via MOs, produces a net gain. Because there is a positive probability that such an opportunity does not arise, having initial cost strictly greater than $\mathbf{b} \cdot \mathbf{S}_{\bar{T}}$ is enough to ensure it is not an arbitrage portfolio. This is due to the fact that if the agent can only execute MOs, the portfolio $\mathbf{b}$ (which costs $\mathbf{b} \cdot \mathbf{S}_{\bar{T}}$ to obtain) can be liquidated for exactly $\mathbf{b} \cdot \mathbf{S}_{\bar{T}}$ only in the best case scenario (on the opposite boundary). In fact, this scenario is only in the closure of $\mathcal{A}$, and hence the mid-price process can only move arbitrarily close to it. Notice that the only assumption we have made here is that the spreads are positive constants.

In the two-dimensional illustrative example consisting of a futures contract and its underlying, we have

$$
\mathcal{A}=\left\{\mathrm{s} \in \mathbb{R}^{2}:\left|s_{1}-s_{2}\right|<\frac{1}{2}\left(\xi_{1}+\xi_{2}\right)\right\}
$$

That is, at any time $t<\bar{T}$, the mid-prices of these two assets cannot differ by more than the sum of their half-spreads; if they do, then one could execute two MOs, for example one order to short the overpriced futures and the other to purchase the underpriced underlying, and hold to maturity for an arbitrage.

The general representation of $\mathcal{A}$ depends on both $\boldsymbol{\xi}$ as well as $B$. More specifically, we show that it depends on the basis vectors of the orthogonal compliment of $B$. The technical details around this are deferred to later in the paper and the representation is given in its dimensionally reduced form, after dimensional reduction in $\mathbf{S}$ (which is far more useful in implementation). However, $\mathcal{A}$ can be represented as a system of linear inequalities (this is made explicit in Equation (24) in the proof of Lemma 9. 
In general, arbitrageurs (who are willing to hold the arbitrage portfolio until $\bar{T}$ ) ensure that prices are always pulled back within the bounds of no-arbitrage. In our framework the investor assumes that for $t \in[0, T]$, the dynamics of the mid-price process $\mathbf{S}_{t}$ is a multivariate reflected and correlated Brownian motion.

Assumption 1. The $n$-dimensional mid-price process $\left\{\mathbf{S}_{t}\right\}_{0 \leq t \leq \bar{T}}$ satisfies

$$
d \mathbf{S}_{t}=\boldsymbol{\sigma} d \mathbf{B}_{t}
$$

for $t \in[0, T]$ and $\mathbf{S}_{t} \in \mathcal{A}$. Here $\boldsymbol{\sigma}$ is an $n$-by-n matrix with $\boldsymbol{\sigma} \boldsymbol{\sigma}^{\prime}$ being positive definite, $\left\{\mathbf{B}_{t}\right\}_{0 \leq t \leq T}$ is a standard Brownian motion on $\mathbb{R}^{n}$, and $\mathbf{S}_{t}$ has (normal) reflection at the boundary of $\mathcal{A}$.

To ensure that normal reflection is well defined, we require $\mathcal{A}$ to be sufficiently regular. However, this poses no technical issues since $\mathcal{A}$ can be represented as a system of linear inequalities and hence, the boundaries are planes in $\mathbb{R}_{n}$ (as shown later in Equation (24)). The resulting boundary is therefore regular ${ }^{1}$ and this is enough for normal reflection to be well defined on the boundary of $\mathcal{A}$. One may think that this is slightly overkill since the only potentially worrisome points in our economy would be the intersection points of these planes, of which there are only finitely many of.

When the risky assets possess a structural dependence, as in our illustrative example, the matrix $\boldsymbol{\sigma}$ will induce a strong correlation between all or some of the $n$ assets. For example, the case discussed above consisting of $n=2$, a futures contract and its underlying, $\sigma$ will induce a strong positive correlation. In general, for assets that exhibit strong structural dependence, $\boldsymbol{\sigma}$ will induce movements in the mid-price processes that are generally in the same direction, on average, to some element of the subspace $B^{\perp}$ (the orthogonal complement of $B$ ).

Recall that the agent will be submitting both LOs and MOs and therefore needs to model incoming MOs from other participants in addition to her own executions (which she controls). As such, she assumes that other market participants send MOs according to independent Poisson Processes. The counting processes representing the total number of MOs submitted by other agents up until time $t$ are $\left\{N_{i, t}^{-}: i=1, \ldots, n\right\}_{0 \leq t \leq T}$ for sells and $\left\{N_{i, t}^{+}: i=\right.$ $1, \ldots, n\}_{0 \leq t \leq T}$ for buys ( $i$ indexes the asset). On the other hand, the total number (including the agent's trades) of market sells and buys up until time $t$ are given by the processes $\left\{\bar{N}_{i, t}^{-}\right.$: $i=1, \ldots, n\}_{0 \leq t \leq T}$ and $\left\{\bar{N}_{i, t}^{+}: i=1, \ldots, n\right\}_{0 \leq t \leq T}$ respectively. It is also convenient to have

\footnotetext{
${ }^{1}$ A point $x \in \mathcal{S} \subset \mathbb{R}_{n}$ (for closed set $\mathcal{S}$ ) is said to be regular if the first hitting time $T=\inf \left\{t>0: B_{t} \in \mathcal{S}\right\}$ satisfies $\mathbb{P}\left[T=0 \mid B_{0}=x\right]=1$. That is, a closed set is regular if a Brownian motion that starts on the boundary immediately re-enters the set with probability 1.
} 
these processes in the following vector forms

$$
\mathbf{N}_{t}^{ \pm}=\left(N_{1, t}^{ \pm}, \ldots, N_{n, t}^{ \pm}\right)^{\prime} \quad \text { and } \quad \overline{\mathbf{N}}_{t}^{ \pm}=\left(\bar{N}_{1, t}^{ \pm}, \ldots, \bar{N}_{n, t}^{ \pm}\right)^{\prime}
$$

where' denotes the transpose operator. Finally, the total number of MOs sent by the highfrequency trader (HFT) up to time $t$ is $\overline{\mathbf{N}}_{t}^{ \pm}-\mathbf{N}_{t}^{ \pm}$.

Thus,

$$
N_{t}=\sum_{i=1}^{n}\left(N_{i, t}^{-}+N_{i, t}^{+}\right)
$$

is the total number of MOs executed by other agents up to time $t$, and

$$
\bar{N}_{t}=\sum_{i=1}^{n}\left(\bar{N}_{i, t}^{-}+\bar{N}_{i, t}^{+}\right)
$$

denotes the total number of MOs executed by all agents up to time $t$. It is also convenient to have these processes in the following vector forms: $\mathbf{N}_{t}=\left(N_{1, t}, \ldots, N_{n, t}\right)^{\prime}$ and $\overline{\mathbf{N}}_{t}=$ $\left(\bar{N}_{1, t}, \ldots, \bar{N}_{n, t}\right)^{\prime}$. Thus, the total number of MOs sent by the investor up to time $t$ is $\bar{N}_{t}-N_{t}$.

The investor sends LOs to the LOB at depth $\delta_{t}^{i}, i=1, \cdots, n$, and we summarize this information in the (2n-dimensional) vector-valued process $\left\{\boldsymbol{\delta}_{t}\right\}_{0 \leq t \leq T}$. Here we use the convention that LO depth is from across the book: the limit buy order is measured from the best ask, and the limit sell order is measured from the best bid quote. Therefore, the investor's buy LOs are posted at $\mathbf{S}_{t}+\boldsymbol{\xi} / 2-\boldsymbol{\delta}_{t}^{-}$, and her sell LOs are posted at $\mathbf{S}_{t}-\boldsymbol{\xi} / 2+\boldsymbol{\delta}_{t}^{+}$. In addition, the running counts of the investor's filled LOs are given by the processes $\left\{M_{i, t}^{+}: i=1, \ldots, n\right\}_{0 \leq t \leq T}$ and $\left\{M_{i, t}^{-}: i=1, \ldots, n\right\}_{0 \leq t \leq T}$ for sell and buy orders, respectively, and we summarize this information in the vector-valued processes $\left\{\mathbf{M}_{t}^{+}\right\}_{0 \leq t \leq T}$ and $\left\{\mathbf{M}_{t}^{-}\right\}_{0 \leq t \leq T}$. This allows the investor to post inside the spread. Inside the spread refers to LOs that improve upon the current best bid or offer.

Assumption 2. The rate of arrival (called the fill rate functions) of MOs which hit/lift the agent's LOs for asset $i=1, \ldots, n$, as measured from "across the book", denoted $h_{i}^{ \pm}(\delta)$, satisfy $h_{i}^{ \pm}(0)<\infty, h_{i}^{ \pm}\left(\xi_{i}\right)=\lambda_{i}^{ \pm}$, and $\lim _{\delta \rightarrow \infty} \delta h_{i}^{ \pm}(\delta)=0$. Furthermore, $h_{i}^{ \pm} \in C^{1}([0, \infty))$ with $h^{\prime}<0$.

The investor gets filled when MOs arrive and reach the level that the LO is posted at. More specifically, the deeper in the book the agent posts, the less frequently she gets filled by a matching MO; a dependence that is captured by the functions $h_{i}^{ \pm}(\delta)$. This is in line with how a number of other authors have modeled optimal postings and fill probabilities as in Ho and Stoll (1981), Avellaneda and Stoikov (Avellaneda and Stoikov), Guéant et al. (2013), Bayraktar and Ludkovski (2014), and Cartea and Jaimungal (2015b), among others. The agent 
is restricted from placing LOs that are through market (i.e., $\delta<0$, which is like submitting an offer lower than the current best bid). Hence, the LO depths $\delta$ are always non-negative. This is not restrictive though since placing an LO through market is equivalent to sending an MO, which the agent is permitted to do via an impulse control (as per the formulation in Section $3)$.

In the real-world, if an incoming $\mathrm{MO}$ is of size that is less than or equal to the available size at the best bid/offer in the LOB, then the MO is filled at the price of the best bid/offer. However, if the size of the MO is larger, which can happen if it is a large sweep or if the liquidity in the order book is thin, then the MO must take liquidity from subsequent levels of the LOB. In this case, the average fill rate of the MO will be worse than the price at the best bid/offer, every LO at the first level will get a fill and some LOs at subsequent levels will also get fills (at their respective limit prices). This is modeled in our fill rate function by a decreasing fill rate as a function of distance from the far side of the LOB.

We may suppress the \pm and $i$ indices on the function $h$ and the scalar variables $\lambda, \kappa$ (defined in Example 7 ), $\delta$, and $\xi$, when it is clear which element is being represented.

Lastly, we provide a few comments on which parameters are and are not observable in the market. The mid-prices $\mathbf{S}$ and spreads $\boldsymbol{\xi}$ are both directly observable in market data. The volatility matrix $\boldsymbol{\sigma}$ is not observable, but is typically estimated via the sample variance on a moving window basis. This can be adjusted when there is a relevant macroeconomic event, e.g., US Federal Reserve Board meetings to set interest rates and US non-farm payrolls data print, both of which are released at predetermined times and generally exhibit very high volatility. The fill rate functions $h_{i}^{ \pm}$are also not observable, but they can be estimated by historical data employing the sizes of incoming MOs and the states of the LOB when the MO are sent, so one can obtain an empirical distribution of fill probabilities. If we take $h$ to be from the exponential family, as in Example 7, then we can fit to the nearest element of this family of functions. The activity rates $\lambda_{i}^{ \pm}$also require estimation and one can proceed as in Cartea et al. (2014) and references therein.

The only other parameter that is not observable is the risk aversion parameter $\phi$. This parameter is specific to every agent. In this context, where the agent can submit MOs, a common technique for calibrating this risk aversion parameter is to ask the agent "given some fixed values for the other parameters (i.e. volatility, spreads, etc), what is the maximum inventory you can tolerate carrying before you pay that spread to reduce?" This will then allow us to back out the value for $\phi$ that is suitable for this agent's risk tolerance (although this will need to be done via iterative search). In practice, this can prove extremely useful and although $\phi$ is calibrated to "usual volatility and liquidity conditions", the same $\phi$ can be used when in "high volatility conditions" since the model will adjust the strategy when presented with a different $\boldsymbol{\sigma}$ matrix. 


\section{The Investor's Optimization Problem}

Recall that $\boldsymbol{\delta}_{t}$ is the depth, as measured from across the book, at which the investor sends quotes to the LOB. Let $(\boldsymbol{\tau} ; \boldsymbol{\varsigma})=\left\{\left(\tau_{1}, \varsigma_{1}\right),\left(\tau_{2}, \varsigma_{2}\right), \ldots\right\}$ be an impulse control representing the investor's submission of MOs to the LOB. The ordered sequence of uniformly bounded $\mathcal{F}_{t}$ stopping times, $0 \leq \tau_{1} \leq \tau_{2} \leq \cdots \leq T$, is the sequence of times of the MOs, and the binary sequence $\varsigma_{1}, \varsigma_{2}, \ldots$ indicates whether the $\mathrm{MO}$ is a buy or a sell order. Recall that the filtration $\left\{\mathcal{F}_{t}\right\}_{0 \leq t \leq T}$ is generated by the processes $\mathbf{S}_{t}, \mathbf{M}_{t}^{ \pm}, \mathbf{N}_{t}^{ \pm}, \overline{\mathbf{N}}_{t}^{ \pm}$.

The cash process $X=\left\{X_{t}\right\}_{0 \leq t \leq T}$ of the investor satisfies the SDE

$$
\begin{aligned}
d X_{t}= & \left(\mathbf{S}_{t}-\boldsymbol{\xi} / 2+\boldsymbol{\delta}_{t-}^{+}\right) \cdot d \mathbf{M}_{t}^{+}-\left(\mathbf{S}_{t}+\boldsymbol{\xi} / 2-\boldsymbol{\delta}_{t-}^{-}\right) \cdot d \mathbf{M}_{t}^{-} \\
& +\left(\mathbf{S}_{t}-\boldsymbol{\xi} / 2\right) \cdot d\left(\overline{\mathbf{N}}_{t}^{-}-\mathbf{N}_{t}^{-}\right)-\left(\mathbf{S}_{t}+\boldsymbol{\xi} / 2\right) \cdot d\left(\overline{\mathbf{N}}_{t}^{+}-\mathbf{N}_{t}^{+}\right),
\end{aligned}
$$

where $\mathbf{S}_{t}, \boldsymbol{\delta}_{t}^{-}, \boldsymbol{\delta}_{t}^{+}$, and $\boldsymbol{\xi}$ are all $n$-dimensional row vectors, and $\mathbf{M}_{t}^{-}, \mathbf{M}_{t}^{+}, \mathbf{N}_{t}^{-}, \mathbf{N}_{t}^{+}, \overline{\mathbf{N}}_{t}^{-}, \overline{\mathbf{N}}_{t}^{+}$ are all $n$-dimensional column vectors.

The terms in the first line in (9) of the cash process account for the changes in cash due to filled limit sell and buy orders, and the terms in the second line account for changes in cash due to the investor's own market sell and buy orders.

The investor seeks the strategy

$$
\left(\left\{\boldsymbol{\delta}_{t} \geq 0\right\}_{0 \leq t \leq T}, \boldsymbol{\tau}, \boldsymbol{\varsigma}\right),
$$

which maximizes her expected terminal wealth while penalizing and constraining inventories. Here $\boldsymbol{\delta}_{t}$ is a bounded $\mathcal{F}_{t}$-previsible process representing the LO quote depths, $\boldsymbol{\tau}$ is an increasing sequence of $\mathcal{F}_{t}$-stopping times (bounded above by $T$ ) representing the times of MO submissions, and $\varsigma$ is the binary sequence of buy/sell indicators. The collection of all such strategies is called the admissible set and denoted by $\mathcal{X}$.

For $0<T \ll \bar{T}$, the investor solves the control problem

$H(t, x, \mathbf{s}, \mathbf{q})=\sup _{\left(\left\{\boldsymbol{\delta}_{t}\right\}_{t}, \boldsymbol{\tau}, \boldsymbol{\varsigma}\right) \in \mathcal{X}} \mathbb{E}_{t, x, \mathbf{s}, \mathbf{q}}\left[X_{T}+\sum_{i=1}^{n} q_{T}^{i}\left(S_{T}^{i}-\operatorname{sign}\left(q_{T}^{i}\right) \frac{\xi_{i}}{2}-\gamma q_{T}^{i}\right)-\phi \int_{t}^{T} \mathbf{q}^{\prime} \boldsymbol{\sigma} \boldsymbol{\sigma}^{\prime} \mathbf{q} d u\right]$

where $\mathbb{E}_{t, x, \mathbf{s}, \mathbf{q}}[\cdot]$ denotes the expectation conditional on $X_{t^{-}}=x, \mathbf{S}_{t}=\mathbf{s}, \mathbf{q}_{t^{-}}=\mathbf{q}$. Here, $\gamma \geq 0$, $\phi \geq 0, \mathbf{q}_{t}=\left(q_{t}^{1}, \ldots, q_{t}^{n}\right)$ is the investor's inventory at time $t$, and $\ell(\cdot) \geq 0$ is an instantaneous inventory penalty. We assume $T \ll \bar{T}$ so that asset prices within the no-arbitrage region $\mathcal{A}$ can be modeled as correlated Brownian motions. Near time $\bar{T}$, the model breaks down, and one must instead employ something akin to a co-integration model which forces the prices to satisfy the zero variance portfolio constraint in Definition 1. 
In Equation (11), we have included two inventory penalties. The first, $q_{T}^{i}\left(-\operatorname{sign}\left(q_{T}^{i}\right) \frac{\xi_{i}}{2}-\gamma q_{T}^{i}\right)$, is a cost that the investor incurs to unwind terminal inventory. To do so the investor uses an MO that crosses the spread, picks up the cost $\xi_{i} / 2$, and walks down one side of the LOB where these costs are given by the linear "market impact" term $\gamma q_{T}^{i}$. The second,

$$
\int_{t}^{T} \mathbf{q}^{\prime} \boldsymbol{\sigma} \boldsymbol{\sigma}^{\prime} \mathbf{q} d u
$$

is a running inventory penalty which allows the agent to control her exposure to inventory risk through time, but is not a financial cost, see Cartea and Jaimungal (2015b) and Cartea et al. (2013). We define the shorthand notation, $\ell(\mathbf{q})$, and represent it as the variance

$$
\ell(\mathbf{q})=\mathbb{V}\left[\mathbf{q} \cdot \widehat{\mathbf{S}}_{1}\right]=\mathbf{q}^{\prime} \boldsymbol{\sigma} \boldsymbol{\sigma}^{\prime} \mathbf{q}
$$

where $\left\{\widehat{\mathbf{S}}_{t}\right\}_{0 \leq t \leq T}$ is the unreflected version of $\left\{\mathbf{S}_{t}\right\}_{0 \leq t \leq T}$.

A priori, it is unclear why the unreflected version of $\mathbf{S}_{t}$ in the penalty function (13) is used. Classical mean-variance portfolio optimization problems penalize according to the variance of the terminal profit and loss (PnL). However, such a penalty fails to characterize how much inventory risk was taken on by the investor throughout the entire life of the strategy. In light of this shortfall, we take the penalty to be the intra-trade quadratic variation of the investor's portfolio, similar to what was done in Forsyth et al. (2012), which is a natural characterization of the total market risk taken on by the investor. The following observation gives a mathematical justification for the use of the inventory penalty in (13).

Observation 5. For $0 \leq u \leq v \leq T$, we have the equivalence:

$$
\int_{u}^{v} \mathbb{V}\left[\mathbf{q}_{t} \cdot \widehat{\mathbf{S}}_{1}\right] d t=\langle\mathbf{q} \cdot \mathbf{S}\rangle_{v}-\langle\mathbf{q} \cdot \mathbf{S}\rangle_{t_{\bar{N}}}+\sum_{k=\bar{N}_{u}+1}^{\bar{N}_{v}}\left\{\langle\mathbf{q} \cdot \mathbf{S}\rangle_{t_{k}-}-\langle\mathbf{q} \cdot \mathbf{S}\rangle_{t_{k-1}}\right\}
$$

where $t_{k}$ is the time of the $k^{\text {th }} M O, t_{k}$ - is the left limit at $t_{k}$, and $\langle\cdot\rangle_{t}$ is the quadratic variation operator. Recall that $\left\{\widehat{\mathbf{S}}_{t}\right\}_{0 \leq t \leq T}$ is the unreflected version of $\left\{\mathbf{S}_{t}\right\}_{0 \leq t \leq T}$.

The relation in (14) is also highlighted in Cartea and Jaimungal (2015b) where the authors offer a similar relation for the one-dimensional case. Thus, the trader penalizes the strategy with the "variance per second" of her current portfolio, which is scaled by $\phi \geq 0$ according to her inventory-risk tolerance. In a single asset economy, this penalty reduces to $\int q_{u}^{2} d u$ (up to a multiplicative constant), which has been shown in Cartea et al. (2013) to stem from ambiguity aversion on the drift of the mid-price process. 


\subsection{Solving the Value Function}

By appealing to Øksendal and Sulem (2007), the value function $H(t, x, \mathbf{s}, \mathbf{q})$, as defined in (11), satisfies the following HJBQVI on the no-arbitrage region $\mathcal{A}$ :

$$
\begin{aligned}
& \max \left[\sup _{\boldsymbol{\delta}>0}\left\{\left(\partial_{t}+\mathcal{L}\right) H+\sum_{i} h_{i}^{-}\left(\delta_{i}^{-}\right) \Delta_{i}^{-} H+\sum_{i} h_{i}^{+}\left(\delta_{i}^{+}\right) \Delta_{i}^{+} H-\phi \ell(\mathbf{q})\right\} ;\right. \\
&\left.\max _{i, \pm}\left\{H\left(t, x \pm s_{i}-\xi_{i} / 2, \mathbf{s}, \mathbf{q} \mp e_{i}\right)-H\right\}\right]=0, \quad \mathbf{s} \in \mathcal{A},
\end{aligned}
$$

with terminal condition $H(T, \cdot)=x+\sum_{i=1}^{n} q_{i}\left(s_{i}-\operatorname{sign}\left(q_{i}\right) \frac{\xi_{i}}{2}-\gamma q_{i}\right)$, the Neumann condition on the boundary of $\mathcal{A}$

$$
\frac{\partial H}{\partial \boldsymbol{n}}=\nabla H \cdot \boldsymbol{n}=0, \quad \forall \mathbf{s} \in \partial \mathcal{A}
$$

where $\boldsymbol{n}$ is the normal vector to $\partial \mathcal{A}$, and where $\mathcal{L}=\frac{1}{2} \sum_{i, j=1}^{n}\left[\boldsymbol{\sigma} \boldsymbol{\sigma}^{\prime}\right]_{i j} \frac{\partial^{2}}{\partial s_{i} \partial s_{j}}$ is the infinitesimal generator of the $n$-dimensional correlated Brownian motion B. Finally, we use $\Delta_{i}^{ \pm}$to denote the difference operator

$$
\Delta_{i}^{ \pm} \varphi(t, x, \mathbf{s}, \mathbf{q})=\varphi\left(t, x \pm s_{i} \pm \delta_{i}^{ \pm} \mp \xi_{i} / 2, \mathbf{s}, \mathbf{q} \mp e_{i}\right)-\varphi(t, x, \mathbf{s}, \mathbf{q})
$$

where $e_{i}$ is a vector with 1 in the $i^{\text {th }}$ position and 0 elsewhere, and dependence on $\delta$ is suppressed for convenience. For example, $\Delta_{i}^{-} \varphi$ denotes the change in $\varphi$ due to the investor's limit buy order being filled: inventory for asset $i$ increases by 1 , and cash $x$ decreases by the cost of the transaction $s_{i}-\delta_{i}^{-}+\xi_{i} / 2$. The interpretation for $\Delta_{i}^{+} \varphi$ when the investor's limit sell order is filled has a similar interpretation.

Lemma 6. If the mid-price process is in the no-arbitrage region (i.e. $\mathbf{s} \in \mathcal{A})$, then the supremum term in (15) is equal to the sum of the supremum over each component.

Proof. The supremum operator satisfies countable sub-additivity. Equality comes from the fact that the $2 n$ variables being optimized appear exactly once in all of $2 n$ distinct additive terms.

We apply the following ansatz for the value function

$$
H(t, x, \mathbf{s}, \mathbf{q})=x+\mathbf{q} \cdot \mathbf{s}+g(t, \mathbf{s}, \mathbf{q})
$$


and recalling that $h(\delta)$ is the fill rate function facing the investor for an LO placed at depth $\delta$, the first order condition for each component of $\boldsymbol{\delta}$ in the continuation region is given by

$$
h^{\prime}\left(\delta_{i}^{ \pm, *}\right)\left(\delta_{i}^{ \pm, *}-\frac{\xi_{i}}{2}+\Delta_{i}^{ \pm} g\right)+h_{i}^{ \pm}\left(\delta_{i}^{ \pm, *}\right)=0
$$

The implicit form in Equation (19) is then used to simplify (15) and obtain a new QVI for $g(\cdot)$

$$
\max \left[\left(\partial_{t}+\mathcal{L}\right) g-\sum_{i, \pm} \frac{\left(h\left(\delta^{*}\right)\right)^{2}}{h^{\prime}\left(\delta^{*}\right)}-\phi \ell(\mathbf{q}), \max _{i, \pm}\left\{\Delta_{i}^{ \pm} g-\xi_{i} / 2\right\}\right]=0
$$

with terminal condition $g(T, \cdot)=-\sum q_{i}\left(\operatorname{sign}\left(q_{i}\right) \frac{\xi_{i}}{2}+\gamma q_{i}\right)$ and boundary condition $(\mathbf{q}+\nabla g)$. $\boldsymbol{n}=0$ on $\partial \mathcal{A}$.

In order to obtain an explicit form for $\boldsymbol{\delta}^{*}$ (in feedback control form), we further assume that $h(\delta)$ is exponential.

Example 7. Exponential Fill Rate. Suppose that MOs arrive to the market according to a Poisson process and the probability of being filled decays exponentially. More precisely, $h_{i}^{ \pm}(\delta)=\lambda_{i}^{ \pm} e^{-\kappa_{i}^{ \pm}\left(\delta-\xi_{i}\right)}$ with $0<\kappa_{i}^{ \pm} \leq 1 / \xi_{i}$.

Example 7 provides an additional modeling assumption on the fill rate facing the investor that will be used in all numerical demonstrations throughout this paper - it is also common for a power law function to be used as the fill rate function. The additional condition that $\kappa_{i}^{ \pm} \leq 1 / \xi_{i}$ is imposed so that a risk-neutral investor following an optimal market making strategy does not post limit quotes inside the spread.

In this exponential fill rate setting, we have

$$
\delta_{i}^{ \pm, *}=\frac{1}{\kappa_{i}^{ \pm}}+\frac{\xi_{i}}{2}-\Delta_{i}^{ \pm} g
$$

and Equation (20), in the continuation region, further reduces to

$$
g_{t}+\frac{1}{2} \sum_{i, j=1}^{n}\left[\boldsymbol{\sigma} \boldsymbol{\sigma}^{\prime}\right]_{i j} \frac{\partial^{2} g}{\partial s_{i} \partial s_{j}}+\sum_{i, \pm} \frac{\lambda_{i}^{ \pm}}{\kappa_{i}^{ \pm}} \exp \left\{-1+\frac{\kappa_{i}^{ \pm} \xi_{i}}{2}+\kappa_{i}^{ \pm} \Delta_{i}^{ \pm} g\right\}-\phi \ell(\mathbf{q})=0 .
$$

The optimal LO depth has two main components. The first is given by the two first terms on the right-hand side in (21) which is the optimal depth chosen by an investor who does not impose any inventory penalty, nor does she pick up any costs for unwinding terminal inventory, 
$\gamma=\phi=0$. The second component, given by the third term on the right-hand of the equation, is a correction to the optimal posting to account for the adjustment to the LO depth as a result of the investor's inventory management strategy.

Proposition 8. The $\max _{i, \pm}\left\{\Delta_{i}^{ \pm} g-\xi_{i} / 2\right\}$ component in the QVI (20) give rise to the LO spread bounds $1 / \kappa_{i}^{ \pm}<\delta_{i}^{ \pm} \leq 1 / \kappa_{i}^{ \pm}+\xi_{i}$ for each $i=1, \ldots, n$.

When considering $\delta^{-}$, the stated lower bound is because the agent is eager to obtain another unit of the asset. Therefore, rather than posting an LO at the strict lower bound of $1 / \kappa_{i}^{ \pm}$, she executes an MO. The upper bound corresponds to posting at a deep level (since the investor does not want the asset), and if the LO is filled, the agent immediately posts an MO to unwind and attempts to make a profit. Profits in this case are not guaranteed and depend on the resilience of the LOB. Note that $\delta_{i}^{-}(\mathbf{q})$ is arbitrarily close to $1 / \kappa_{i}^{ \pm}$if and only if $\delta_{i}^{+}\left(\mathbf{q}+e_{i}\right)$ is close to $1 / \kappa_{i}^{ \pm}+\xi_{i}$ and similarly, $\delta_{i}^{+}(\mathbf{q})$ is arbitrarily close to $1 / \kappa_{i}^{ \pm}$if and only if $\delta_{i}^{-}\left(\mathbf{q}-e_{i}\right)$ is close to $1 / \kappa_{i}^{ \pm}+\xi_{i}$.

\section{Viscosity Solution and Numerical Scheme for Exponential Fill Rate}

This section outlines the technical details regarding the existence of a unique viscosity solution to the dynamic programming equation (DPE), specifies a dimensional reduction in the spatial variables, gives explicit details regarding the discrete numerical scheme, and proves that the discretized value function converges to the unique viscosity solution.

\subsection{Dimensional Reduction of Spatial Variables}

We first prove the following lemma to demonstrate a dimensional reduction in the spatial variable $\mathbf{s}$. Due to this lemma, the relevant $\mathbf{s}$ values live in a compact domain. For a fixed error, the total computational run time will then be proportional to $c^{\operatorname{dim} B}(2 Q+1)^{n}$, where $c$ is some constant that depends on the spreads $\xi_{1}, \xi_{2}, \ldots, \xi_{n}$, the basis vectors that span $B$ (or equivalently on the number of discrete steps on the dimensionally reduced s-grid), and $Q$ is the maximum inventory the investor can be long or short for each asset.

Lemma 9. There exists a $\mathcal{A}^{\prime}$ such that $\overline{\mathcal{A}}^{\prime} \subset \mathbb{R}^{n^{\prime}}, n^{\prime}=\operatorname{dim} B$, and a $C^{2}$, surjective function $\Gamma: \overline{\mathcal{A}} \longrightarrow \overline{\mathcal{A}}^{\prime}$ such that

(I) For all $\mathbf{s} \in \overline{\mathcal{A}}, g(\mathbf{s}, \cdot)=g((\Gamma(\mathbf{s}), \mathbf{0}), \cdot)$, 
(II) $\mathbf{s} \in \partial \mathcal{A} \Longleftrightarrow \Gamma(\mathbf{s}) \in \partial \mathcal{A}^{\prime}$

(III) $\overline{\mathcal{A}}^{\prime}$ is the cartesian product of closed, bounded intervals.

Proof. Define $m:=\operatorname{dim} B$. Then $B=\operatorname{span}\left\{b_{1}, \ldots, b_{m}\right\}$, where $b_{1}, \ldots, b_{m}$ is an orthogonal basis, and for each $i=1, \ldots, m$ we have $\left(\right.$ wlog) $\mathbb{P}\left[b_{i} \cdot \mathbf{S}_{\bar{T}}=0\right]=1$. No arbitrage (i.e. $\mathbf{s} \in \mathcal{A}$ ) is equivalent to the system of inequalities

$$
\mathbf{y} \cdot \mathbf{s}+\sum_{j=1}^{n}\left|y_{j}\right| \frac{\xi_{j}}{2}>0 \quad \text { for } \mathbf{y}=\left( \pm b_{1}, \ldots, \pm b_{m}\right)^{\prime}
$$

which simplifies to

$$
-\sum_{j=1}^{n}\left|y_{j}\right| \frac{\xi_{j}}{2}<y \cdot \mathbf{s}<\sum_{j=1}^{n}\left|y_{j}\right| \frac{\xi_{j}}{2} \quad \text { for } \mathbf{y}=\left(b_{1}, \ldots, b_{m}\right)^{\prime}
$$

Because the $b_{i}$ 's are linearly independent, one can see that (24) is equivalent to $\Gamma(\mathbf{s})$ being inside some rectangular subset of $\mathbb{R}^{m}$ for the function $\Gamma(\mathbf{s})=\left[b_{j} \cdot \mathbf{s}\right]_{j}$, which is also clearly $C^{2}$. At this point, (II) should be obvious from (24) and the definition of $\Gamma,(I I I)$ is proven, and linear independence of the basis vectors gives $(I V)$.

To prove $(I)$, we must appeal to some invariance properties of $g$. Notice that $\overline{\mathcal{A}}$ can now be reparameterized to the product space

$$
\prod_{i=1}^{m}\left[-\sum_{j=1}^{n}\left|b_{i} \cdot e_{j}\right| \frac{\xi_{j}}{2}, \sum_{j=1}^{n}\left|b_{i} \cdot e_{j}\right| \frac{\xi_{j}}{2}\right] \times \mathbb{R}^{n-m}
$$

From equation (20) and the Neumann boundary condition (only depends on s via the direction of the normal vector, which only depends on which piece of the boundary $\mathbf{s}$ is on; this can be determined uniquely via $\Gamma(\mathbf{s})$ by taking a limiting sequence in $\mathbf{s}$ to the boundary), we see that under this new parameterization, $g$ only depends on $\mathbf{s}$ through the first $m$ components. Hence, if $\mathbf{s}_{1}, \mathbf{s}_{2} \in \overline{\mathcal{A}}$ are such that $\Gamma\left(\mathbf{s}_{1}\right)=\Gamma\left(\mathbf{s}_{2}\right)$, then $g\left(\mathbf{s}_{1}\right)=g\left(\mathbf{s}_{2}\right)$ and $(I)$ is proved.

Lemma 9 states that it is not necessary to know $\mathbf{s}$ to obtain the value of $g(\mathbf{s}, \cdot)$; we only require the information contained in $\Gamma(\mathbf{s})$. Furthermore, there exists such a function $\Gamma$ that 
provides a nice, smooth mapping between the original $n$-dimensional no-arbitrage region $\overline{\mathcal{A}}$ and the new $n^{\prime}$-dimensional no-arbitrage region $\overline{\mathcal{A}}^{\prime}$, and that this new region is a bounded rectangle. The fact that the new region is bounded improves the accuracy of the numerical scheme discussed below in Section 4.2 since we no longer have to approximate the unbounded set $\overline{\mathcal{A}}$ by a bounded one. There is, however, a minor book-keeping detail. The form $(\Gamma(\mathbf{s}), \mathbf{0})$, where $\mathbf{0}$ is an $\left(n-n^{\prime}\right)$-dimensional vector of 0 's, requires the assets to be ordered in a certain way. In an arbitrary ordering, the components of $(\Gamma(\mathbf{s}), \mathbf{0})$ will need to be re-ordered. We continue with the labels that produce a simplification of this form as it is more compact to write $(\Gamma(\mathbf{s}), \mathbf{0})$ instead of having the 0 's in arbitrary positions (i.e. not consecutive nor all at the beginning or end).

In some sense, $\Gamma(\mathbf{s})$ is sufficient for $g .{ }^{2}$ That is, $\Gamma(\mathbf{s})$ contains the same amount of information as $\mathbf{s}$ when computing $g$. Furthermore, one could say that $\Gamma(\mathbf{s})$ is minimal sufficient since it can be written as a function of any other function of $\mathbf{s}$ that is sufficient for $g$. Stated heuristically, $\Gamma(\mathbf{s})$ contains the smallest amount of information about $\mathbf{s}$ that still allows us to know $g(\mathbf{s})$ with certainty. The construction of $\Gamma$ in the proof of Lemma 9 is used in what follows to obtain a dimensional reduction, and is given by

$$
\Gamma(\mathbf{s})=\left[b_{j} \cdot \mathbf{s}\right]_{j}
$$

In our two-dimensional illustrative example, we take $\Gamma(\mathbf{s})=s_{1}-s_{2}$. For the 3 -dimensional example with two nearby futures and the 1-2 calendar spread, one could take $\Gamma(\mathbf{s})=s_{1}-s_{2}-s_{3}$. More details for these two examples are provided in Appendix A.

The following Corollary states that the function $g$ is invariant to movements in the direction of any element of $B^{\perp}$. This would correspond to vectors of the form $(1,1)$ in our illustrative example (i.e. $g$ is remains unchanged when the futures and its underlying both increase or decrease by exactly the same amount).

Corollary 10. $D_{v} g=0$ on $\overline{\mathcal{A}}$ whenever $v \in B^{\perp}$.

Proof. Let $\mathbf{s} \in \overline{\mathcal{A}}$ and $v \in B^{\perp}$. Note that we necessarily have $\mathbf{s}+v \in \overline{\mathcal{A}}$ by Equation (24) since $b_{i} \cdot v=0$ for $i=1, \ldots, n^{\prime}$. We then have $\Gamma(\mathbf{s})=\Gamma(\mathbf{s}+v)$ by $(26)$. But $v \in B^{\perp}$ is arbitrary and $B^{\perp}$ is a subspace, so one can consider a limiting sequence of the form $\left\{\eta_{k} v\right\}$, with the scalar sequence $\eta_{k} \downarrow 0$, which yields $D_{v} g=0$.

\footnotetext{
${ }^{2}$ In mathematical statistics, a statistic $U$ is said to be sufficient for an unknown model parameter $\Theta$ if the conditional distribution of the data, conditional on $U$, is independent of $\Theta$. Heuristically speaking, $U$ contains the same amount of information about $\Theta$ as the data itself.
} 


\subsection{Description of Numerical Scheme}

We numerically solve (22) by applying upper and lower bounds to each component in $\mathbf{q}$, and solving the corresponding PDEs backwards in time using an explicit numerical scheme. For any fixed $\mathbf{q}$, we have an $n$-dimensional discrete grid to solve for various values of $\mathbf{s}$. Once we have this grid for each $\mathbf{q}$, we propagate one step backwards in $t$ and repeat the process.

Define the domain for $\mathbf{s}, \mathcal{A}^{\prime} \subset \mathbb{R}^{n^{\prime}}$, on the dimensionally reduced grid. Let $\mathbb{T}_{d t}$ be a regular partition of $[0, T], \mathbb{S}_{d s}$ be an $n^{\prime}$-dimensional regular rectangular partition of the dimensionally reduced s-grid $\overline{\mathcal{A}}^{\prime}$, and $\mathbb{Y}_{Q}$ be the subset of $\mathbb{Z}^{n}$ that corresponds to inventory with maximum $Q$ as previously described (i.e. $\mathbb{Y}_{Q}=\mathbb{Z}^{n} \cap[-Q, Q]^{n}$ ). Throughout the rest of this paper, $d t>0$ and $d s>0$ refer to the discrete step sizes in the time $(t)$ and space $(\Gamma(\mathbf{s}))$ variables, respectively.

Before we proceed, we also define the covariance matrix, $\widetilde{\boldsymbol{\Sigma}}=\widetilde{\boldsymbol{\sigma}} \widetilde{\boldsymbol{\sigma}}^{\prime}$, of the dimensionally reduced diffusion process (as per Section 4.1).

Define, for any function $\varphi:[0, T] \times \overline{\mathcal{A}}^{\prime} \times \mathbb{Z}^{n} \longrightarrow \mathbb{R}$, the discrete dynamic evolution operator

$$
\mathcal{S}^{d t, d s, Q}(t, \mathbf{s}, \mathbf{q}, \varphi)=\max \left[\mathcal{T}^{d t, d s, Q}(t, \mathbf{s}, \mathbf{q}, \varphi), \mathcal{M}^{d t, d s, Q}(t, \mathbf{s}, \mathbf{q}, \varphi)\right],
$$

with the component corresponding to the continuation region given by

$$
\begin{gathered}
\mathcal{T}^{d t, d s, Q}(t, \mathbf{s}, \mathbf{q}, \varphi)=\varphi(\cdot)+d t\left[\frac{1}{2} \sum_{i, j=1}^{n^{\prime}}\left[\tilde{\boldsymbol{\sigma}} \widetilde{\boldsymbol{\sigma}}^{\prime}\right]_{i j} \widehat{\mathcal{T}}_{i j}^{d t, d s, Q}(\cdot, \varphi)+\sum_{i, \pm} \frac{\lambda}{\kappa e} \exp \left\{\frac{\kappa \xi}{2}+\kappa \Delta \varphi(\cdot)\right\}-\phi \ell(\mathbf{q})\right] \\
\widehat{\mathcal{T}}_{i j}^{d t, d s, Q}(t, \mathbf{s}, \mathbf{q}, \varphi)=\frac{\varphi\left(\Gamma\left(s_{i}+d s, s_{j}+d s\right), \cdot\right)-\varphi\left(\Gamma\left(s_{i}+d s\right), \cdot\right)-\varphi\left(\Gamma\left(s_{j}+d s\right), \cdot\right)+\varphi(\cdot)}{d s^{2}}
\end{gathered}
$$

where $\varphi(\cdot)$ is shorthand for $\varphi(t, \mathbf{s}, \mathbf{q})$, and the component corresponding to the impulse region given by

$$
\mathcal{M}^{d t, d s, Q}(t, \mathbf{s}, \mathbf{q}, \varphi)=\max _{i, \pm}\left\{\varphi\left(t, \mathbf{s}, \mathbf{q} \pm e_{i}\right)-\xi_{i} / 2\right\}
$$

Note that the standard finite difference is used in $\widehat{\mathcal{T}}_{i j}^{d t, d s, Q}(t, \mathbf{s}, \mathbf{q}, \varphi)$ when $i=j$.

We approximate the solution $g$ by $g^{d t, d s, Q}: \mathbb{T}_{d t} \times \mathbb{S}_{d s} \times \mathbb{Y}_{Q} \longrightarrow \mathbb{R}$ defined by:

$$
\left\{\begin{aligned}
g^{d t, d s, Q}(T, \mathbf{s}, \mathbf{q}) & =-\sum q_{i}\left(\operatorname{sign}\left(q_{i}\right) \frac{\xi_{i}}{2}+\gamma q_{i}\right) \\
g^{d t, d s, Q}(d t \cdot k, \mathbf{s}, \mathbf{q}) & =\mathcal{S}^{d t, d s, Q}\left(d t \cdot(k+1), \mathbf{s}, \mathbf{q}, g^{d t, d s, Q}\right) .
\end{aligned}\right.
$$

We employ an explicit scheme backwards in time as follows. 
(1) When $t=T$, for each $\mathbf{s}$ and $\mathbf{q}$ on their respective grids, set

$$
g^{d t, d s, Q}(T, \mathbf{s}, \mathbf{q})=-\sum q_{i}\left(\operatorname{sign}\left(q_{i}\right) \frac{\xi_{i}}{2}+\gamma q_{i}\right) .
$$

(2) For $t<T$ and $\mathbf{s} \in \mathcal{A}^{\prime}$, we perform the following: Compute $\mathcal{T}^{d t, d s, Q}\left(t+d t, \mathbf{s}, \mathbf{q}, g^{d t, d s, Q}\right)$ and store the optimal control $\delta$. Some details for handling the mixed partial term are given in Appendix A.1. Then compute $\mathcal{M}^{d t, d s, Q}\left(t+d t, \mathbf{s}, \mathbf{q}, g^{d t, d s, Q}\right)$ and store the optimal impulse $\tau$. If $\mathcal{T}^{d t, d s, Q}\left(t+d t, \mathbf{s}, \mathbf{q}, g^{d t, d s, Q}\right) \geq \mathcal{M}^{d t, d s, Q}\left(t+d t, \mathbf{s}, \mathbf{q}, g^{d t, d s, Q}\right)$, then set

$g^{d t, d s, Q}(t, \mathbf{s}, \mathbf{q})=\mathcal{T}^{d t, d s, Q}\left(t+d t, \mathbf{s}, \mathbf{q}, g^{d t, d s, Q}\right)$. Otherwise, set

$g^{d t, d s, Q}(t, \mathbf{s}, \mathbf{q})=\mathcal{M}^{d t, d s, Q}\left(t+d t, \mathbf{s}, \mathbf{q}, g^{d t, d s, Q}\right)$.

(3) For $t<T$ and $\mathbf{s} \in \partial \mathcal{A}^{\prime}$, we perform numerically approximate the condition $(\mathbf{q}+\nabla g) \cdot \boldsymbol{n}=0$, similar to what is done in Appendix A.3 and Appendix A.4 for the $n=2$ and $n=3$ examples, respectively.

\subsection{Convergence of the Numerical Scheme}

We require the following bounds on the value function to ensure the existence of a unique viscosity solution to the DPE.

Lemma 11. There exists constants $c_{0}, c_{1}, c_{2} \in \mathbb{R}$ such that

$$
x+\sum_{i} q_{i}\left(s_{i}-\operatorname{sign}\left(q_{i}\right) \frac{\xi_{i}}{2}\right) \leq H \leq x+\mathbf{q} \cdot \mathbf{s}+c_{0}(T-t)+c_{1}\|\mathbf{q}\|_{1}+c_{2} .
$$

Proof. The lower bound is attained by the strategy that immediately liquidates and then 
does nothing until time $T$. For the upper bound we have

$$
\begin{aligned}
H \leq & \left.\sup _{(\boldsymbol{\delta}, \boldsymbol{\tau}) \in \mathcal{U}} \mathbb{E}_{t}\left[X_{T}+\mathbf{q}_{T} \cdot \mathbf{S}_{T}-\phi \int_{t}^{T} \ell\left(\mathbf{q}_{u}\right) d u\right]\right|_{\mathbf{q}=0}+\mathbf{q} \cdot \mathbf{s}+\|\mathbf{q}\|_{1} \sum_{i} \xi_{i} \\
\leq & \left.\sup _{(\boldsymbol{\delta}, \boldsymbol{\tau}) \in \mathcal{U}^{\prime}} \mathbb{E}_{t}\left[X_{T}+\mathbf{q}_{T} \cdot \mathbf{S}_{T}-\phi \int_{t}^{T} \ell\left(\mathbf{q}_{u}\right) d u\right]\right|_{\mathbf{q}=0}+\mathbf{q} \cdot \mathbf{s} \\
& +(T-t)\left(\sum_{i} \xi_{i}+\sup _{\delta, i, \pm} \delta h(\delta)\right)+\|\mathbf{q}\|_{1} \sum_{i} \xi_{i} \\
= & +\sum_{i} \xi_{i} \sup _{\omega, t, i}\left(\mathbf{q}_{t} \cdot e_{i}\right) c_{3}(\sigma) \sqrt{T-t} \mathbb{E}\left[\chi_{1}\right]+(T-t) \sum_{i} \xi_{i} \sup _{\delta, i, \pm} \delta h(\delta) \\
& +\mathbf{q} \cdot \mathbf{s}+(T-t)\left(\sum_{i} \xi_{i}+\sup _{\delta, i, \pm} \delta h(\delta)\right)+\|\mathbf{q}\|_{1} \sum_{i} \xi_{i},
\end{aligned}
$$

where $\chi_{u}$ is the maximum (in absolute value) of a standard $n$-dimensional Brownian motion up to time $u$ and $\mathcal{U}^{\prime} \subset \mathcal{U}$ is the subset of admissible controls with $\boldsymbol{\delta}=\infty$ whenever $\|\mathbf{q}\|=0$. Finally, the result follows from Assumption 2.

Although this upper bound is not necessarily sharp, it is sufficient to continue. We now turn our attention to each term in the upper bound in this proof. The terms $\mathbf{q} \cdot \mathbf{s}+\|\mathbf{q}\|_{1} \sum_{i} \xi_{i}$ correspond to free liquidation to $\mathbf{q}=0$ with an added bonus of $\sum_{i} \xi_{i}$ per unit of each open position. The investor cannot do better than this (or even come close) on an expected value basis. The term

$$
(T-t)\left(\sum_{i} \xi_{i}+\sup _{\omega, t, i, \pm} \delta h(\delta)\right)
$$

is an upper bound on the profit of all future open positions that use filled LOs. Lastly, we must consider all MO initiated open positions (for the case where $\mathbf{q}=0$ is not an element of the continuation region). If these positions are aided with filled LOs, then we have already accounted for such events. Otherwise, in order to produce a profit, the Brownian motion must travel a certain distance from where the open position was initiated. The total number of times this happens is proportional to $\sqrt{T-t}$, and $\phi>0$ guarantees that $\mathbf{q}_{t}(\omega)$ is uniformly bounded. We are able to bound the expected round trip profit because the dimensionally reduced no-arbitrage region is bounded. 
Similar to Guilbaud and Pham $(2015)^{3}$, we first prove monotonicity, stability, and consistency properties (Propositions 12, 13, and 14, respectively) of the discrete dynamic evolution operator $\mathcal{S}^{d t, d s, Q}(t, \mathbf{s}, \mathbf{q}, \cdot)$. These properties are critical in the proof of Theorem 15, which states that the solution to the numerical scheme, as given by (31), converges locally uniformly to the unique viscosity solution $g(t, \mathbf{s}, \mathbf{q})$.

We also impose the stricter condition that $\widetilde{\boldsymbol{\Sigma}}$ is diagonal as to guarantee that the scheme is monotonic when working in higher dimensions $\left(n^{\prime}>1\right)$. Note that all the examples provided in this paper, as well as common applications in practice, adhere to this restriction even though we have $n>1$. This is a direct benefit of the dimensional reduction in Section 4.1.

Proposition 12. (Monotonicity) Suppose $\widetilde{\boldsymbol{\Sigma}}$ is diagonal. For any $d s>0$, there exists $h_{t}>0$ (depends on ds) such that for any $0<d t<h_{t}, Q \in \mathbb{N}$, and $\varphi_{1}, \varphi_{2} \in C_{b}^{1,2}\left([0, T] \times \overline{\mathcal{A}}^{\prime} \times \mathbb{Z}^{n}\right)$ with $\varphi_{1} \leq \varphi_{2}$, we have $\mathcal{S}^{d t, d s, Q}\left(t, \mathbf{s}, \mathbf{q}, \varphi_{1}\right) \leq \mathcal{S}^{d t, d s, Q}\left(t, \mathbf{s}, \mathbf{q}, \varphi_{2}\right)$.

Proof. Let $\varphi_{1} \leq \varphi_{2}, Q \in \mathbb{N}$, and $d s>0$. Monotonicity of the operator $\mathcal{M}$ is obvious.

To prove monotonicity of the operator $\mathcal{T}$, we rely on the dimensional reduction in Lemma 9. We remark that we require monotonicity only on the restricted lattice where $0<d t<h_{t}$. Also, we suppress the arguments $(t, \mathbf{s}, \mathbf{q})$ of the functions $\varphi$. to help with the comprehension of the proof.

Because $\varphi$ and its derivatives are continuous, and the domain of $\varphi$ is compact, there exists a finite constant $A_{1}>0$ (the compactness of $\overline{\mathcal{A}}^{\prime}$ is critical for this existence) such that

$$
e^{\kappa \Delta \varphi_{2}}-e^{\kappa \Delta \varphi_{1}} \geq\left(e^{-\kappa \varphi_{2}}-e^{-\kappa \varphi_{1}}\right) e^{\kappa \mathbb{S} \varphi_{1}}=\left(e^{-\kappa\left(\varphi_{2}-\varphi_{1}\right)}-1\right) e^{\kappa \Delta \varphi_{1}} \geq-A_{1}\left(\varphi_{2}-\varphi_{1}\right),
$$

where $A_{1}$ does not depend on $\varphi_{1}$ or $\varphi_{2}$, and $\mathbb{S}$ is the shift operator associated with $\Delta$.

Next we note that the diffusion approximation term greatly reduces by the assumption that

\footnotetext{
${ }^{3}$ Although our problem is more involved than that of Guilbaud and Pham (2015) because we have a diffusion term that plays an important role, this is discussed in the details of Propositions 12 to 14.
} 
$\widetilde{\Sigma}$ is diagonal. More specifically,

$$
\hat{\mathcal{T}}_{i j}^{d t, d s, Q}\left(t, \mathbf{s}, \mathbf{q}, \varphi_{2}\right)-\hat{\mathcal{T}}_{i j}^{d t, d s, Q}\left(t, \mathbf{s}, \mathbf{q}, \varphi_{1}\right) \geq \frac{-2\left(\varphi_{2}-\varphi_{1}\right)}{d s^{2}} \mathbf{1}_{\{i=j\}}
$$

Hence, we obtain the bound

$$
\frac{1}{2} \sum_{i, j=1}^{n^{\prime}}\left[\widetilde{\boldsymbol{\sigma}} \widetilde{\boldsymbol{\sigma}}^{\prime}\right]_{i j} \widehat{\mathcal{T}}_{i j}^{d t, d s, Q}(\cdot, \varphi) \geq-A_{2} \frac{\varphi_{2}-\varphi_{1}}{d s^{2}}
$$

where $A_{2}>0$ depends on $\widetilde{\boldsymbol{\Sigma}}$, but is free of $d s$.

Using (35) and (37) gives

$$
\begin{aligned}
\mathcal{T}^{d t, d s, Q}\left(t, \mathbf{s}, \mathbf{q}, \varphi_{2}\right)- & \mathcal{T}^{d t, d s, Q}\left(t, \mathbf{s}, \mathbf{q}, \varphi_{1}\right) \\
= & \varphi_{2}-\varphi_{1}+d t \frac{1}{2} \sum_{i, j=1}^{n^{\prime}}\left[\tilde{\boldsymbol{\sigma}} \widetilde{\boldsymbol{\sigma}}^{\prime}\right]_{i j}\left(\hat{\mathcal{T}}_{i j}^{d t, d s, Q}\left(\cdot, \varphi_{2}\right)-\hat{\mathcal{T}}_{i j}^{d t, d s, Q}\left(\cdot, \varphi_{1}\right)\right) \\
& \quad+d t \sum_{i, \pm} \frac{\lambda}{\kappa} e^{-1+\kappa \xi / 2}\left(e^{\kappa \Delta \varphi_{2}}-e^{\kappa \Delta \varphi_{1}}\right) \\
\geq\left(\varphi_{2}-\varphi_{1}\right) & {\left[1-d t\left\{A_{1} \sum_{i, \pm} \frac{\lambda}{\kappa} e^{-1+\kappa \xi / 2}+\frac{A_{2}}{d s^{2}}\right\}\right] . }
\end{aligned}
$$

Taking

$$
d t<\left(A_{1} \sum_{i, \pm} \frac{\lambda}{\kappa} e^{-1+\kappa \xi / 2}+\frac{A_{2}}{d s^{2}}\right)^{-1}
$$

gives monotonicity of $\mathcal{T}$.

Proposition 13. (Stability) For any $d t, d s>0$ and $Q \in \mathbb{N}$, there exists a unique solution $g^{d t, d s, Q}(t, \mathbf{s}, \mathbf{q})$ to (31). Furthermore, the sequence $\left\{g^{d t, d s, Q}\right\}$ is uniformly bounded. There exist $c_{0}, c_{1}, c_{2} \in \mathbb{R}$ such that for any $(t, \mathbf{s}, \mathbf{q}) \in \mathbb{T}_{d t} \times \mathbb{S}_{d s} \times \mathbb{Y}_{Q}$,

$$
-\sum_{i} q_{i} \operatorname{sign}\left(q_{i}\right) \frac{\xi_{i}}{2} \leq g^{d t, d s, Q}(t, \mathbf{s}, \mathbf{q}) \leq c_{0}(T-t)+c_{1}\|\mathbf{q}\|_{1}+c_{2}
$$


Proof. Existence and uniqueness follows immediately from the definition of the explicit discretization scheme given in (31). The upper bound is a direct consequence of Lemma 11 since $g^{d t, d s, Q}(t, \mathbf{s}, \mathbf{q}) \leq g(t, \mathbf{s}, \mathbf{q})=H-x-\mathbf{q} \cdot \mathbf{s} \leq c_{0}(T-t)+c_{1}\|\mathbf{q}\|_{1}+c_{2}$. The lower bound is attained by immediate liquidation of the current inventory and then doing nothing until $t=T$.

Proposition 14. (Consistency) For all $(t, \mathbf{s}, \mathbf{q}) \in[0, T) \times \overline{\mathcal{A}}^{\prime} \times \mathbb{Z}^{n}$ and $\varphi \in C_{b}^{1,2}\left([0, T] \times \overline{\mathcal{A}}^{\prime} \times \mathbb{Z}^{n}\right)$, we have

$$
\begin{aligned}
\lim _{(d t, d s, Q) \rightarrow(0,0, \infty)} & \frac{1}{d t}\left[\mathcal{T}^{d t, d s, Q}\left(t^{\prime}+d t, \mathbf{s}^{\prime}, \mathbf{q}, \varphi\right)-\varphi\left(t^{\prime}, \mathbf{s}^{\prime}, \mathbf{q}\right)\right]= \\
\left(t^{\prime}, \mathbf{s}^{\prime}\right) \rightarrow(t, \mathbf{s}) & \\
& \frac{\partial \varphi}{\partial t}+\frac{1}{2} \sum_{i, j=1}^{n^{\prime}}\left[\widetilde{\boldsymbol{\sigma}} \widetilde{\boldsymbol{\sigma}}^{\prime}\right]_{i j} \frac{\partial^{2} \varphi}{\partial s_{i} \partial s_{j}}+\sum_{i, \pm} \frac{\lambda}{\kappa} \exp \left\{-1+\frac{\kappa \xi}{2}+\kappa \Delta \varphi\right\}-\phi \ell(\mathbf{q})
\end{aligned}
$$

and

$$
\begin{aligned}
& \lim _{(d t, d s, Q) \rightarrow(0,0, \infty)} \mathcal{M}^{d t, d s, Q}\left(t^{\prime}+d t, \mathbf{s}^{\prime}, \mathbf{q}, \varphi\right)=\max _{i, \pm}\left\{\Delta_{i}^{ \pm} \varphi-\xi_{i} / 2\right\} \\
& \left(t^{\prime}, \mathbf{s}^{\prime}\right) \rightarrow(t, \mathbf{s})
\end{aligned}
$$

Proof. Equation (41) follows by continuity of $\varphi$. To prove $(40)$, let $\left(t^{\prime}, \mathbf{s}^{\prime}, \mathbf{q}\right) \in[0, T) \times \overline{\mathcal{A}}^{\prime} \times \mathbb{Z}^{n}$ and observe

$$
\begin{aligned}
\frac{1}{d t}\left[\mathcal{T}^{d t, d s, Q}\left(t^{\prime}+d t, \mathbf{s}^{\prime}, \mathbf{q}, \varphi\right)-\varphi\left(t^{\prime}, \mathbf{s}^{\prime}, \mathbf{q}\right)\right] & =\frac{1}{d t}\left[\varphi\left(t^{\prime}+d t, \mathbf{s}^{\prime}, \mathbf{q}\right)-\varphi\left(t^{\prime}, \mathbf{s}^{\prime}, \mathbf{q}\right)\right]-\phi \ell(\mathbf{q}) \\
& +\frac{1}{2} \sum_{i, j=1}^{n^{\prime}}\left[\widetilde{\boldsymbol{\sigma}} \widetilde{\boldsymbol{\sigma}}^{\prime}\right]_{i j} \hat{\mathcal{T}}_{i j}^{d t, d s, Q}\left(t^{\prime}+d t, \mathbf{s}^{\prime}, \mathbf{q}, \varphi\right) \\
& +\sum_{i, \pm} \frac{\lambda}{\kappa} \exp \left\{-1+\frac{\kappa \xi}{2}+\kappa \Delta \varphi\left(t^{\prime}+d t, \mathbf{s}^{\prime}, \mathbf{q}\right)\right\}
\end{aligned}
$$

The only term that does not trivially converge is $\Delta \varphi\left(t^{\prime}+d t, \mathbf{s}^{\prime}, \mathbf{q}\right.$ ) (the other terms clearly converge since $\left.\varphi \in C_{b}^{1,2}\right)$. But $\Delta \varphi\left(t^{\prime}+d t, \mathbf{s}^{\prime}, \mathbf{q}\right)$ is also continuous in $(t, \mathbf{s})$ whenever $Q \geq$ $\|\mathbf{q}\|_{\infty}+1$, thus proving (40).

Observe that we do not need to include a sequence of inventory levels due to the fact that $\mathbf{q}$ resides on the sparse grid $\mathbb{Z}^{n}$. One could only consider the limiting sequence $\left(t^{\prime}+d t, \mathbf{s}^{\prime}, \mathbf{q}^{\prime}\right) \rightarrow$ 
$(t, \mathbf{s}, \mathbf{q})$ sufficiently far in the tail when $\mathbf{q}^{\prime}=\mathbf{q}$ onwards.

Theorem 15. (Convergence) Suppose $\widetilde{\boldsymbol{\Sigma}}$ is diagonal. The solution $g^{d t, d s, Q}$ to the numerical scheme (31) converges locally uniformly to the unique viscosity solution $g(t, \mathbf{s}, \mathbf{q})$ on $[0, T) \times$ $\overline{\mathcal{A}}^{\prime} \times \mathbb{Z}^{n}$, as $(d t, d s, Q) \rightarrow(0,0, \infty)$, provided $d t<f(d s)$ for some $f: \mathbb{R}^{+} \rightarrow \mathbb{R}^{+}$.

Proof. Follows from Propositions 12, 13, 14, and Barles and Souganidis (1991). For completeness, the details are provided.

The limits

$$
\begin{aligned}
& g_{*}(t, \mathbf{s}, \mathbf{q}):= \liminf _{(d t, d s, Q)} \rightarrow(0,0, \infty) \\
&\left(t^{\prime}, \mathbf{s}^{\prime}\right) \rightarrow(t, \mathbf{s}) \\
& g^{*}(t, \mathbf{s}, \mathbf{q}):=\quad g^{d t, d s, Q}\left(t^{\prime}, \mathbf{s}^{\prime}, \mathbf{q}\right), \\
& \quad \limsup _{(d t, d s, Q)} \rightarrow(0,0, \infty) \\
&\left(t^{\prime}, \mathbf{s}^{\prime}\right) \rightarrow(t, \mathbf{s})
\end{aligned}
$$

are lower/upper semi-continuous functions on $[0, T] \times \overline{\mathcal{A}}^{\prime} \times \mathbb{Z}^{n}$, and inherit the boundedness of $\left\{g^{d t, d s, Q}\right\}$ by the stability result in Proposition 13. It suffices to show (by symmetry and the strong comparison principle) that $g_{*}$ possesses the viscosity supersolution property. To this end, let $(\bar{t}, \overline{\mathbf{s}}, \overline{\mathbf{q}}) \in[0, T) \times \overline{\mathcal{A}}^{\prime} \times \mathbb{Z}^{n}$ and $\varphi \in C_{b}^{1,2}\left([0, T] \times \overline{\mathcal{A}}^{\prime} \times \mathbb{Z}^{n}\right)$ such that $(\bar{t}, \overline{\mathbf{s}}, \overline{\mathbf{q}})$ attains the strict global minimum of $g_{*}-\varphi$. Then there exists sequences $\left\{\left(t_{k}^{\prime}, \mathbf{s}_{k}^{\prime}, \mathbf{q}_{k}^{\prime}\right)\right\}_{k}$ in $[0, T) \times \overline{\mathcal{A}}^{\prime} \times \mathbb{Z}^{n}$ and $\left\{\left(d t_{k}, d s_{k}, Q_{k}\right)\right\}_{k}$ such that

$$
\begin{aligned}
\left(t_{k}^{\prime}, \mathbf{s}_{k}^{\prime}, \mathbf{q}_{k}^{\prime}\right) & \longrightarrow(\bar{t}, \overline{\mathbf{s}}, \overline{\mathbf{q}}), \\
\left(d t_{k}, d s_{k}, Q_{k}\right) & \longrightarrow(0,0, \infty), \\
g^{d t_{k}, d s_{k}, Q_{k}} & \longrightarrow g_{*}(\bar{t}, \overline{\mathbf{s}}, \overline{\mathbf{q}}),
\end{aligned}
$$

and $\left(t_{k}^{\prime}, \mathbf{s}_{k}^{\prime}, \mathbf{q}_{k}^{\prime}\right)$ is the global minimizer of $g^{d t_{k}, d s_{k}, Q_{k}}-\varphi$. To apply the monotonicity result in Proposition 12 , the sequence $\left\{\left(d t_{k}, d s_{k}, Q_{k}\right)\right\}_{k}$ must have $d t_{k}$ sufficiently small for each $d s_{k}$, as prescribed by (38). 
Denote the sequence of minimum values $\left\{\beta_{k}\right\}_{k}$ by $\beta_{k}:=\left(g^{d t_{k}, d s_{k}, Q_{k}}-\varphi\right)\left(t_{k}^{\prime}, \mathbf{s}_{k}^{\prime}, \mathbf{q}_{k}^{\prime}\right)$. Then we have the relation $g^{d t_{k}, d s_{k}, Q_{k}} \geq \varphi+\beta_{k}$. Applying the definition of the numerical scheme in (31) and the monotonicity result from Proposition 12 gives:

$$
\begin{aligned}
\beta_{k}+\varphi\left(t_{k}^{\prime}, \mathbf{s}_{k}^{\prime},\right. & \left.\mathbf{q}_{k}^{\prime}\right)=g^{d t_{k}, d s_{k}, Q_{k}}\left(t_{k}^{\prime}, \mathbf{s}_{k}^{\prime}, \mathbf{q}_{k}^{\prime}\right) \\
& =\mathcal{S}^{d t_{k}, d s_{k}, Q_{k}}\left(t_{k}^{\prime}+d t_{k}, \mathbf{s}_{k}^{\prime}, \mathbf{q}_{k}^{\prime}, g^{d t_{k}, d s_{k}, Q_{k}}\right) \\
& \geq \mathcal{S}^{d t_{k}, d s_{k}, Q_{k}}\left(t_{k}^{\prime}+d t_{k}, \mathbf{s}_{k}^{\prime}, \mathbf{q}_{k}^{\prime}, \varphi+\beta_{k}\right) \\
& =\mathcal{S}^{d t_{k}, d s_{k}, Q_{k}}\left(t_{k}^{\prime}+d t_{k}, \mathbf{s}_{k}^{\prime}, \mathbf{q}_{k}^{\prime}, \varphi\right)+\beta_{k} \\
& =\max \left[\mathcal{T}^{d t_{k}, d s_{k}, Q_{k}}\left(t_{k}^{\prime}+d t_{k}, \mathbf{s}_{k}^{\prime}, \mathbf{q}_{k}^{\prime}, \varphi\right), \mathcal{M}^{d t_{k}, d s_{k}, Q_{k}}\left(t_{k}^{\prime}+d t_{k}, \mathbf{s}_{k}^{\prime}, \mathbf{q}_{k}^{\prime}, \varphi\right)\right]+\beta_{k}
\end{aligned}
$$

We then have the relation

$$
\begin{aligned}
\min \left\{\frac{1}{d t_{k}}\left[\varphi\left(t_{k}^{\prime}, \mathbf{s}_{k}^{\prime}, \mathbf{q}_{k}^{\prime}\right)-\mathcal{T}^{d t_{k}, d s_{k}, Q_{k}}\left(t_{k}^{\prime}+d t_{k}, \mathbf{s}_{k}^{\prime}, \mathbf{q}_{k}^{\prime}, \varphi\right)\right]\right. \\
\left.\varphi\left(t_{k}^{\prime}, \mathbf{s}_{k}^{\prime}, \mathbf{q}_{k}^{\prime}\right)-\mathcal{M}^{d t_{k}, d s_{k}, Q_{k}}\left(t_{k}^{\prime}+d t_{k}, \mathbf{s}_{k}^{\prime}, \mathbf{q}_{k}^{\prime}, \varphi\right)\right\} \geq 0,
\end{aligned}
$$

and applying the consistency result in Proposition 14 while taking $k \rightarrow \infty$ finally yields the viscosity supersolution property

$$
\begin{array}{r}
\min \left[-\frac{\partial \varphi}{\partial t}-\frac{1}{2} \sum_{i, j=1}^{n^{\prime}}\left[\tilde{\boldsymbol{\sigma}} \tilde{\boldsymbol{\sigma}}^{\prime}\right]_{i j} \frac{\partial^{2} \varphi}{\partial s_{i} \partial s_{j}}-\sum_{i, \pm} \frac{\lambda}{\kappa} \exp \left\{-1+\frac{\kappa \xi}{2}+\kappa \Delta \varphi\right\}+\phi \ell(\mathbf{q})\right. \\
\left.\varphi-\max _{i, \pm}\left\{\Delta_{i}^{ \pm} \varphi-\frac{\xi_{i}}{2}\right\}\right]\left.\right|_{(\bar{t}, \overline{\mathbf{s}}, \overline{\mathbf{q}})} \geq 0
\end{array}
$$

\section{Optimal Trading: no-arbitrage region and performance of strategy}

\subsection{No-Arbitrage region}

We use our two-dimensional illustrative example to explore the behavior of the optimal strategy. Recall that the first asset $S_{t}^{1}$ is a traded equity and the second asset, $S_{t}^{2}$, is a $\bar{T}$-maturity futures contract written on $S_{t}^{1}$. In this economy, we have $\mathbb{V}\left[(1,-1)^{\prime} \cdot \mathbf{S}_{\bar{T}}\right]=0$, yielding a 
market that is over-complete. To this end, we take $\mathbf{b}=(1,-1)^{\prime}$ as the basis of $B$ for this illustrative example, and other parameters are given in Table 1.

\begin{tabular}{cc}
\hline \hline Parameter & Value \\
\hline$\phi$ & 1 \\
$\sigma$ & $\left(\begin{array}{cc}0.005 & 0.009 \\
0 & 0.015\end{array}\right)$ \\
$\gamma$ & 0.001 \\
$\xi_{1}, \xi_{2}$ & 0.02 \\
$\lambda_{1}^{-}, \lambda_{1}^{+}$ & 1.2 \\
$\lambda_{2}^{-}, \lambda_{2}^{+}$ & 1.0 \\
$\kappa_{1}^{-}, \kappa_{1}^{+}$ & 40 \\
$\kappa_{2}^{-}, \kappa_{2}^{+}$ & 25 \\
$T-t$ & 60 \\
\hline \hline
\end{tabular}

Table 1: Model parameters used in the analysis of the function $g(t, \mathbf{s}, \mathbf{q})$, the continuation region, and the optimal inventory level. Recall that the fill rate assumption $h_{i}^{ \pm}(\delta)=\lambda_{i}^{ \pm} e^{-\kappa_{i}^{ \pm}\left(\delta-\xi_{i}\right)}$ from Example 7 is being made.

For simplicity, we assume that the fill rates on both sides of the LOB are the same (see Table 1) and that the arrival of buy and sell MOs are also the same, but both the fill rate parameter and arrival of MOs differ across the two assets. For example, the underlying equity $S_{t}^{1}$ is modeled with a higher rate of incoming MOs than $S_{t}^{2}$ which is the $\bar{T}$-maturity futures contract. This is because the underlying trades more actively than the first nearby futures and so we assume that there is more liquidity in the equity than the futures by taking $\kappa_{1}^{ \pm}>\kappa_{2}^{ \pm}$. Although this is true for equities, there are cases (such as commodities) when a future's underlying does not actively trade or is a non-traded asset (e.g., electricity).

Finally, note that because $\xi_{i}>0$, we do not require their correlation to be unity or even for their volatilities to be the same. However, it is financially sound for their volatilities to be similar and for their correlation to be positive. This leads to our choice of volatility matrix, $\boldsymbol{\sigma}$, which induces a correlation of approximately 0.87 . The total volatility of Asset 1 turns out to be less than that of Asset 2. The rationale behind this is that the sources of quadratic variation of the futures contract not only include the underlying equity (Asset 1), but also with that of interest rates and stochastic volatility (which affects the futures price via the convexity correction). So the futures contract should then have a larger total volatility than its underlying.

For a fixed time $t$, Figures $1(\mathrm{a})$ and $1(\mathrm{c})$ show $g(t, \mathbf{s}, \mathbf{q})$ as a function of $\mathbf{q}$ for fixed $\mathbf{s}=$ $(100,100)$. It is clear that it attains its maximum at $\mathbf{q}=(0,0)$. The asymmetry in $g$ with respect to inventory is due to the fact that the risk associated with a single unit of Asset 1 is less than that of Asset 2. Furthermore, portfolios of the form $(a, b)$ with $\operatorname{sign}(a) \neq \operatorname{sign}(b)$ are less risky than portfolios with $\operatorname{sign}(a)=\operatorname{sign}(b)$ as the two assets are strongly positively 


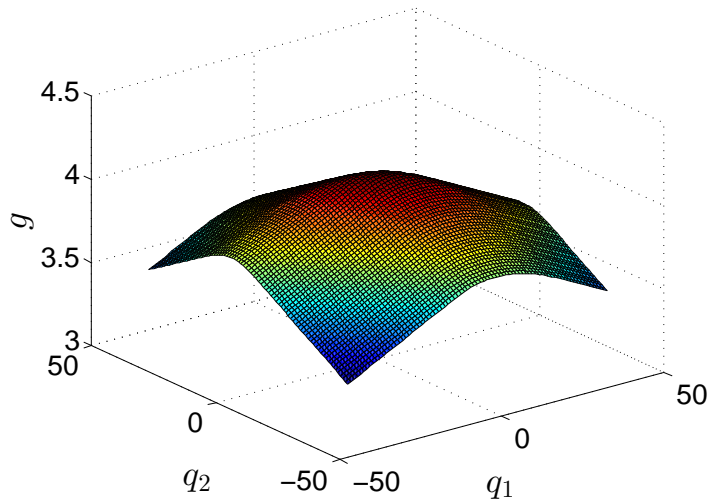

(a) $s_{1}=s_{2}$

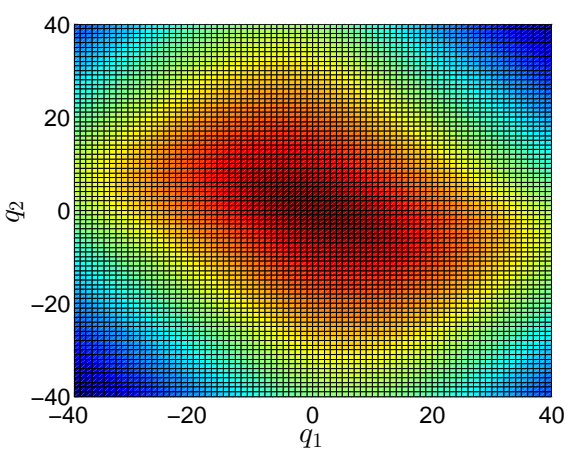

(c) $s_{1}=s_{2}$

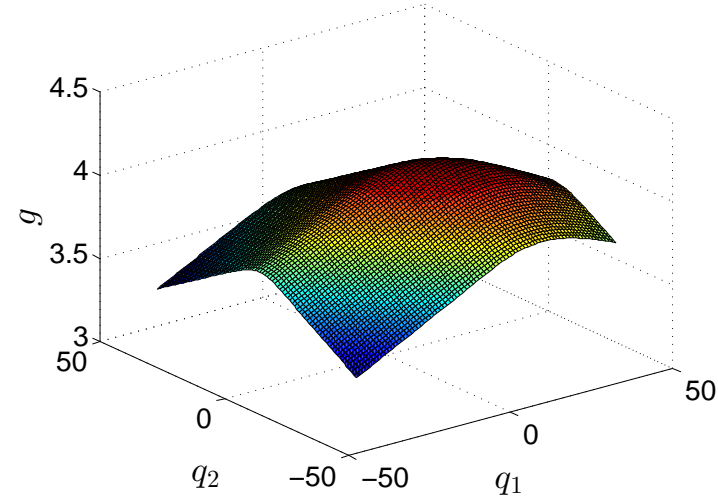

(b) $s_{1}<s_{2}$

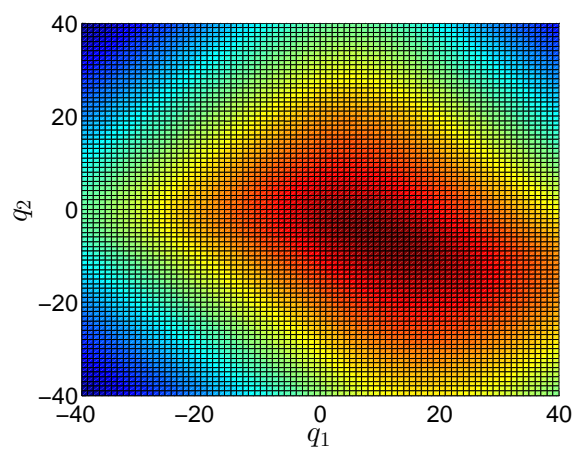

(d) $s_{1}<s_{2}$

Figure 1: $g$ surface for various s. Figures 1(a) and 1(c) show the surface and heatmap, respectively, of $g(t, \mathbf{s}, \mathbf{q})$ when the mid-prices of assets are equal. Figures $1(\mathrm{~b})$ and $1(\mathrm{~d})$ show the surface and heatmap, respectively, of $g(t, \mathbf{s}, \mathbf{q})$ when Asset 1 is cheap relative to Asset $2\left(s_{1}=s_{2}-0.012\right)$.

correlated. This is depicted in the dark blue regions in the upper right and lower left corners of Figure 1(c), which corresponds to large long positions and large short positions in both assets, respectively.

The left and right panels of Figure 1 show $g(t, \mathbf{s}, \mathbf{q})$ as a function of $\mathbf{q}$ for two different values of $s_{1}-s_{2}$. When $s_{1}=s_{2}$, the maximum of $g(t, \mathbf{s}, \mathbf{q})$ is again obtained at $\mathbf{q}=(0,0)$. However, this is no longer the case when $s_{1} \neq s_{2}$. When $s_{1}<s_{2}$, the investor would rather be long Asset 1 and short Asset 2, and she will post LOs in a way that reflects this desire to hold such an inventory. This is because the process $S_{t}^{1}-S_{t}^{2}$ is bounded from below by the sum of the half-spreads of the assets. Hence, when this process is near the lower boundary, there is the potential for profits as we have assumed reflection on this boundary.

Figure 2 shows how the continuation region also depends on $s_{1}-s_{2}$. When $s_{1}=s_{2}$, the continuation region is centered around the origin, just as one would expect. However, when 


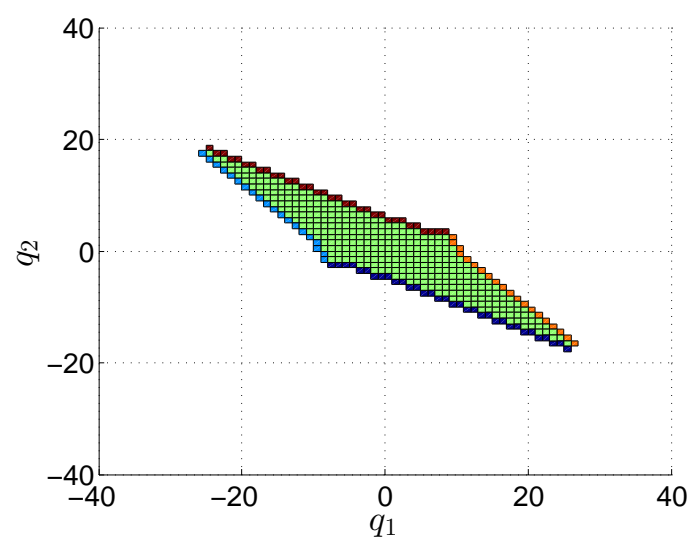

(a) $s_{1}=s_{2}$

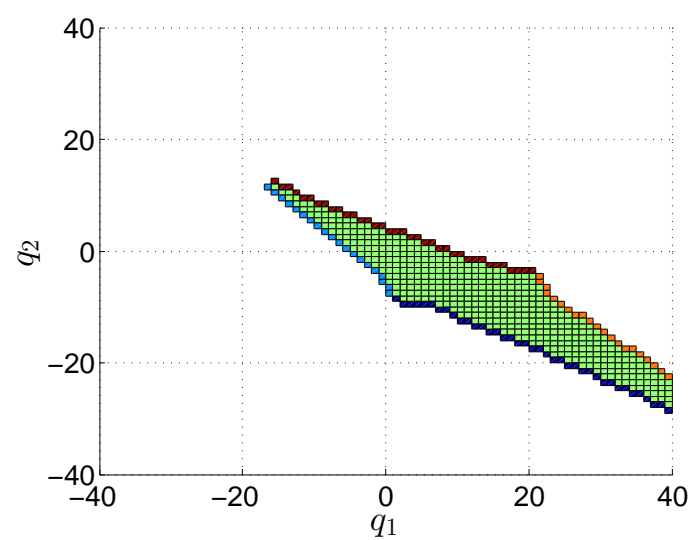

(b) $s_{1}<s_{2}$

Figure 2: Continuation region for various s. The different coloured regions correspond to different MO submissions. Namely, light blue: Asset 1 buy MO; orange: Asset 1 sell MO; dark blue: Asset 2 buy MO; burgundy: Asset 2 sell MO; green: continuation region; and white: unattainable. Recall that Figure 2(b) is specified by $s_{1}=s_{2}-0.012$.

one asset is cheap relative to the other, i.e. $s_{1}<s_{2}$, then the agent prefers to long Asset 1 and short Asset 2 in an attempt to profit when Asset 1 increases relative to Asset 2. Furthermore, she will be aggressive and execute MOs if she is caught holding a portfolio that is short Asset 1 and long Asset 2 when this shift in the mid-prices occurs, as demonstrated by the absence of the continuation region in the upper left corner of Figure 2(b). As the price discrepancy becomes larger, the investor adjusts her strategy to accumulate inventory in a way that positions her to profit from these market conditions.

As discussed earlier, inventory control plays a critical role in investment strategies. The investor actively tries to liquidate outstanding long positions and cover outstanding short positions. In a single asset scenario, the logistics behind this are clear. However, in a multiple asset setting with correlated assets, the investor may attempt to partially hedge a long position in one asset with a short position in another asset that is positively correlated.

Corollary 16. Mean Reversion of Inventory. Given the optimal strategy $\boldsymbol{\delta}^{*}$, the expected drift of the inventory process $\mathbf{q}_{t}$ inside the continuation region is given by

$$
\lim _{u \rightarrow t^{+}} \frac{1}{u-t} \mathbb{E}\left[\mathbf{q}_{u}-\mathbf{q}_{t} \mid \mathcal{F}_{t}\right]=\left[\lambda_{i}^{-} h_{i}^{-}\left(\delta_{i, t}^{-}\right)-\lambda_{i}^{+} h_{i}^{+}\left(\delta_{i, t}^{+}\right)\right]_{i}
$$

ProOF. By observing that each element of $\boldsymbol{\delta}_{t}$ is a right continuous with left limits (RCLL) function of $t$ (for fixed $\omega \in \Omega$ ), we can conclude that the expected drift in inventories inside 


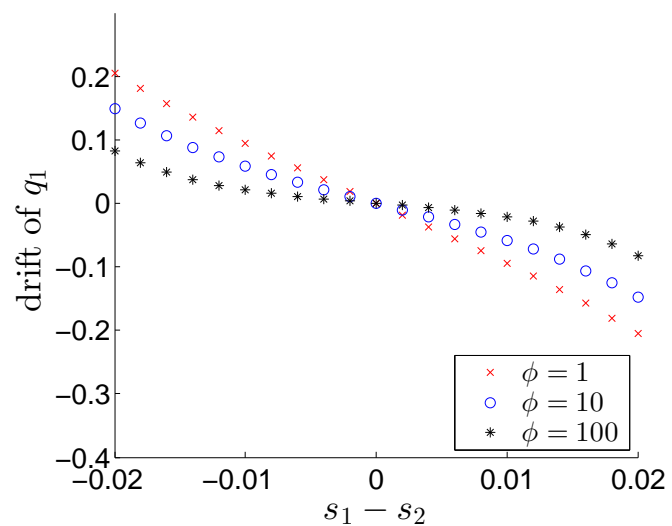

(a) Asset 1

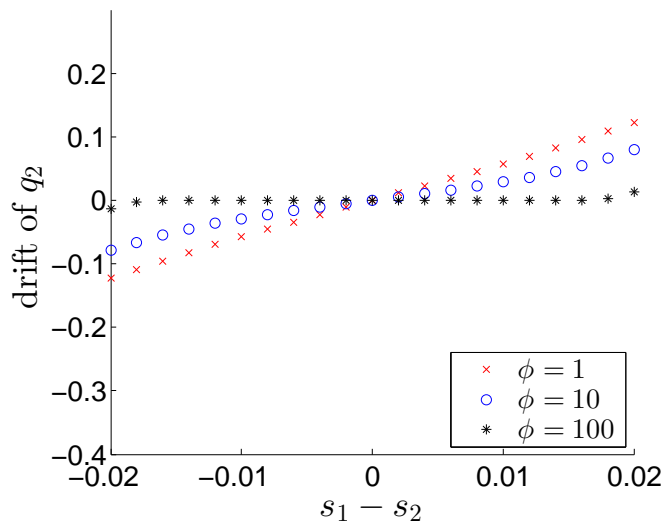

(b) Asset 2

Figure 3: Inventory drift when the investor is flat (i.e. $q_{1}=q_{2}=0$ ).

the continuation region is given by the difference in the arrival rates of filled LOs at time $t$.

Figure 3 shows the inventory drift as a function of the mid-price $\mathbf{s}$. When the trader is flat (holding zero inventory) and Asset 1 is cheap relative to Asset 2 (i.e. when $s_{1}-s_{2}$ is small), she wants to acquire a long position in Asset 1 and short position in Asset 2. The corresponding drifts in the $q_{t}^{1}$ and $q_{t}^{2}$ processes become more pronounced as the inventory penalty parameter $\phi$ decreases.

Figures 4 and 5 show the drift in the process $\mathbf{q}_{t}$ as a function of the current inventory $\mathbf{q}$ for fixed values of $s_{1}-s_{2}$ (and $\phi=1$ as taken in previous plots where not explicitly stated). The inventory will be attracted to the curve where the reversion rate is 0 (colored green). Notice how in each figure the $q_{i}$ process is attracted to a curve and not a point. More specifically, for each value of $\mathbf{q}$, the component $q_{i}$ is attracted to a particular point that depends on the values of the other components of $\mathbf{q}$. When $q_{1}=0$ and $s_{1}=s_{2}$, then the optimal amount of Asset 2 to hold is 0. However, when $q_{1}>0$, then the optimal amount of Asset 2 to hold is less than 0 . This highlights the behaviour that the investor tries to partially hedge a long position in one asset by acquiring a short position in the other (as well as try to reduce the long position in Asset 1 as well).

The intersection point of the corresponding attraction curves for $q_{1}$ and $q_{2}$ then acts as the attraction point for the vector-valued inventory process $\mathbf{q}_{t}$. Figure 6 is a graphical depiction of this attraction point as a function of $s_{1}-s_{2}$. 


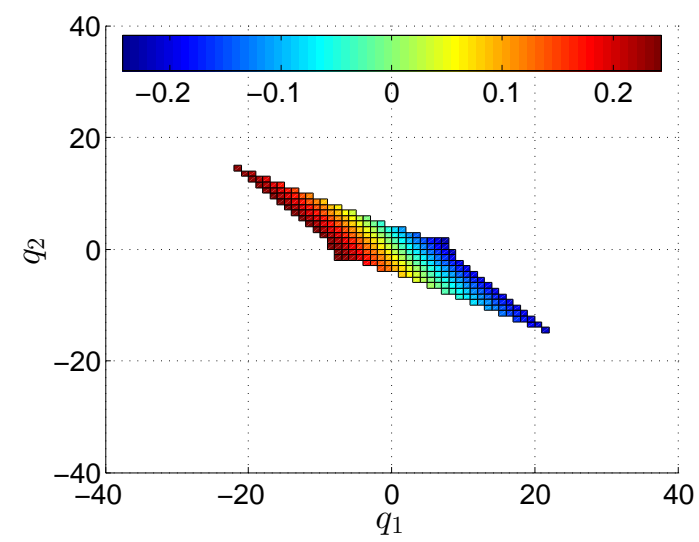

(a) Asset 1

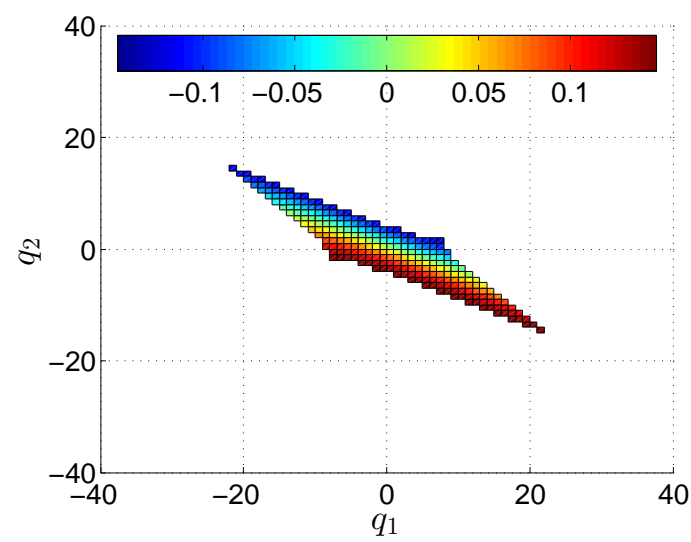

(b) Asset 2

Figure 4: Inventory drift when the mid-prices of the assets are equal (i.e. $s_{1}=s_{2}$ ).

\subsection{Simulation Study: PnL of the Strategy}

In this section we use simulations to analyze the financial performance of an investor who follows the optimal strategy in the last section. We use the time limiting strategy for $T-t$ large since the strategy becomes time invariant fairly quickly (within 15-30 seconds for the collection of parameters we tested). The investor's operating frequency is every 5 milliseconds, the maximum amount of inventory the investor can hold long or short is $Q=40$ for each asset, and the trading horizon is $T=300$ seconds. Other model parameters are provided in Table 2 . Recall that we continue with the fill rate assumption $h_{i}^{ \pm}(\delta)=\lambda_{i}^{ \pm} e^{-\kappa_{i}^{ \pm}\left(\delta-\xi_{i}\right)}$ from Example 7. The bound on the investor's inventory only plays a role in the numerical solution of the value function and was not imposed in the optimal control problem above, but it is trivial to include it. Depending on the penalty parameter $\phi>0$, the exact strategy will dictate uniform (over time and $\Omega$ ) bounds on the investor's inventory since the continuation region is bounded, as argued in Section 4.3. This is visually depicted in Figure 2 where the optimal strategy keeps inventory inside the rhombus shaped region.

\subsection{Facing Adverse Selection}

Here we analyze the strategy under more realistic scenarios, in particular we assume that an important source of risk faced by the investor is adverse selection. The investor might be trading with better informed counterparties and therefore faces the risk of buying assets right before prices drop or selling assets right before prices rise. In our analysis, we include adverse selection by assuming that some MOs will affect the mid-price of the asset, but the investor does not account for this in her model.

With this in mind, we suppose that an MO originates from one of two sources. It was either 


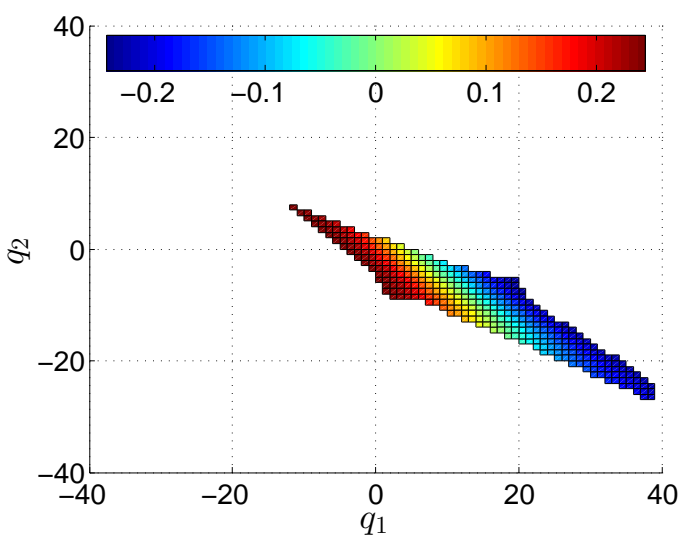

(a) Asset 1

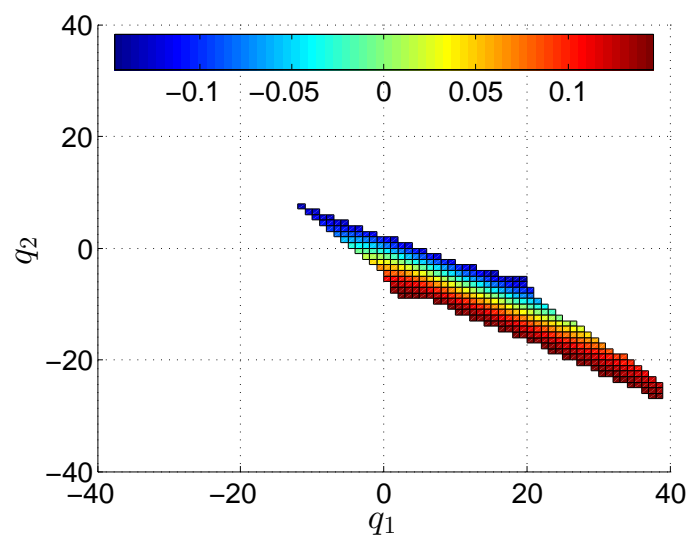

(b) Asset 2

Figure 5: Inventory drift when Asset 1 is cheap relative to Asset 2 (i.e. $s_{1}=s_{2}-0.012$ ).

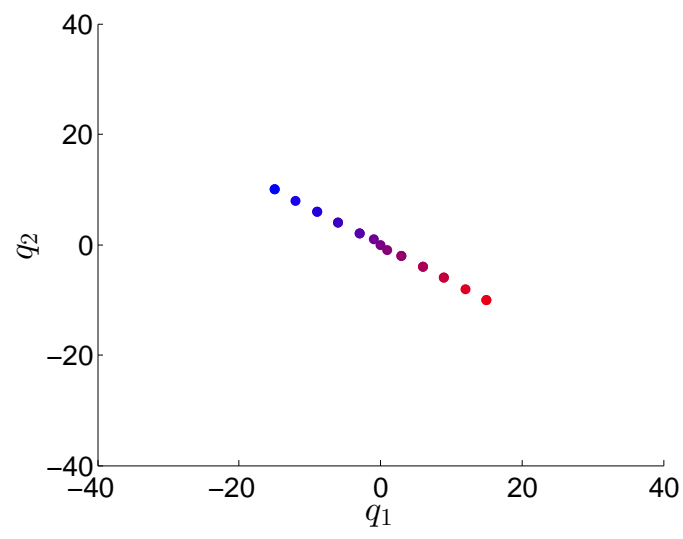

Figure 6: Mean reversion point of $\mathbf{q}$ as a function of $s_{1}-s_{2}$. The red (blue) dots correspond to small (large) values of $s_{1}-s_{2}$.

executed by an informed trader, or an uninformed one. We assume that the probability that any particular MO comes from an informed trader is constant, and we shall denote this probability by $\rho$. We describe the two possible effects by example. Suppose the current best bid-ask quotes for Assets 1 and 2 are 100.00/100.02 and 100.01/100.03, respectively. A large buy MO on Asset 1 arrives, widening the spread to 100.00/100.06. At this point, one of two things will happen immediately.

1) The MO was executed by an informed trader: The best bid-ask quotes for Asset 1 becomes $100.04 / 100.06$, giving an immediate impact of $\$ 0.04$. This impacts Asset 2 in the same way causing the BBO to move to $100.05 / 100.07$. The informed trader knows that there will be upward pressure on the price, and some other informed market participants (on both assets) move their current quotes to reflect this. 


\begin{tabular}{cc}
\hline \hline Parameter & Value \\
\hline$T$ & 300 \\
$\phi$ & $0.3,1,3,10,30,100,200$ \\
$\sigma$ & $\left(\begin{array}{cc}0.005 & 0.009 \\
0 & 0.015\end{array}\right)$ \\
$\gamma$ & 0.001 \\
$\xi_{1}, \xi_{2}$ & 0.02 \\
$\lambda_{1}^{-},, \lambda_{1}^{+}$ & 1.2 \\
$\lambda_{2}^{-}, \lambda_{2}^{+}$ & 1.0 \\
$\kappa_{1}^{-}, \kappa_{1}^{+}$ & 40 \\
$\kappa_{2}^{-}, \kappa_{2}^{+}$ & 25 \\
$\boldsymbol{\zeta}$ & $\left(\begin{array}{cc}2 & 0 \\
0 & 2\end{array}\right)$ \\
$\rho$ & $0.40,0.50,0.60$ \\
$\epsilon$ & $0,0.01,0.02,0.04$ \\
\hline \hline
\end{tabular}

Table 2: Model parameters used in the simulation study. Recall that the fill rate assumption $h_{i}^{ \pm}(\delta)=$ $\lambda_{i}^{ \pm} e^{-\kappa_{i}^{ \pm}\left(\delta-\xi_{i}\right)}$ from Example 7 is being made.

2) The MO was executed by an uninformed trader: The best bid-ask quotes for both assets remains unchanged. There is nothing special about this $\mathrm{MO}$, and the depleted portion of the LOB refills with LOs.

This describes the immediate effect on the mid-price process which now follows

$$
\begin{aligned}
& d \mathbf{S}_{t}=\boldsymbol{\alpha}_{t} d t+\boldsymbol{\sigma} d \mathbf{B}_{t}+\mathfrak{d}_{\left(N_{t-}+1\right)}\left(\begin{array}{cc}
1 & 1 \\
1 & 1
\end{array}\right) d\left(\widehat{\mathbf{N}}_{t}^{+}+\widehat{\mathbf{N}}_{t}^{-}\right) \\
& d \boldsymbol{\alpha}_{t}=-\boldsymbol{\zeta} \boldsymbol{\alpha}_{t} d t+\mathfrak{e}_{\left(N_{t-}+1\right)} \Psi d\left(\widehat{\mathbf{N}}_{t}^{+}-\widehat{\mathbf{N}}_{t}^{-}\right) .
\end{aligned}
$$

Here the vector-valued processes $\left\{\widehat{\mathbf{N}}_{t}^{-}\right\}_{0 \leq t \leq T}$ and $\left\{\widehat{\mathbf{N}}_{t}^{+}\right\}_{0 \leq t \leq T}$ are the total number of informed market sell and buy orders up to time $t$, respectively, with the $i^{\text {th }}$ component representing Asset $i$. The process $\boldsymbol{\alpha}_{t}$ denotes the persistent impact of an informed MO which affects the drift of the mid-price process. Because all of these effects occur simultaneously (or faster than the HFT can respond), this model does not admit latency arbitrage. $\mathbf{S}_{t}$ has (normal) reflection at the boundary of $\mathcal{A}, \boldsymbol{\zeta}$ and $\Psi$ are $n \times n$ matrices with non-negative entries, $\mathfrak{d}_{(1)}, \mathfrak{d}_{(2)}, \ldots$ is the sequence of random variables representing immediate market impact (will be non-negative for market buy orders and non-positive for market sell orders), and $\mathfrak{e}_{(1)}, \mathfrak{e}_{(2)}, \ldots$ is an i.i.d. sequence of exponential random variables with mean 1.

Note that because the immediate market impact term adds a random multiple of $(1,1)^{\prime}$ to $\mathbf{S}_{t}$, the resulting value of the mid-price vector is guaranteed to remain inside the no-arbitrage 
region $\mathcal{A}$. Recall from Section 2 that

$$
\mathcal{A}=\left\{\mathrm{s} \in \mathbb{R}^{2}:\left|s_{1}-s_{2}\right|<\frac{1}{2}\left(\xi_{1}+\xi_{2}\right)\right\}
$$

and $\alpha_{t}^{1}=\alpha_{t}^{2}$, which will be denoted $\alpha_{t}$. Equation (48) is the natural multivariate extension of the drift dynamics presented in Cartea et al. (2014).

When an informed buy MO arrives on Asset $i$, then there will immediately be a jump in $\boldsymbol{\alpha}_{t}$ equal to the $i^{\text {th }}$ column of $\Psi$ multiplied by a random exponential random variable. The matrix $\boldsymbol{\zeta}$ serves as the decay matrix which forces each component of the process $\boldsymbol{\alpha}_{t}$ to mean revert to zero. The effect of an informed sell MO is similar, except it induces a negative jump in $\boldsymbol{\alpha}_{t}$.

Observe that we are using $\widehat{\mathbf{N}}_{t}^{ \pm}$instead of $\mathbf{N}_{t}^{ \pm}$or $\overline{\mathbf{N}}_{t}^{ \pm}$in (48). This has the interpretation that only the MOs of informed traders will have an impact on $\boldsymbol{\alpha}_{t}$. Furthermore, our own MOs do not move the market, unlike the informed traders' MOs, as our MOs are quite small in size and we are a small player without superior market information. For the purposes of illustration, we make the further assumption that

$$
\Psi=\epsilon\left(\begin{array}{ll}
1 & 1 \\
1 & 1
\end{array}\right),
$$

where $\epsilon \geq 0$ is a scale parameter.

Figure 7 shows sample paths for the mid-price process $\mathbf{S}_{t}$ and its drift process $\boldsymbol{\alpha}_{t}$. Observe the jumps in both the mid-price process and in the short-term drift component due to the activity of informed traders. On this particular time window, Asset 1 is priced lower than Asset 2. Observe in Figure 7(c) how the mid-price spends most of the time near the upper boundary. It will be shown in Section 4.1 that $g$ is only a function of $\mathbf{s}$ through the function $s_{1}-s_{2}$. We provide a sample path of this process in Figure $7(\mathrm{~d})$. Again, notice that on this particular window $S_{t}^{(1)}-S_{t}^{(2)}<0$, but still obeying the bound $S_{t}^{(1)}-S_{t}^{(2)}>-\left(\xi_{1}+\xi_{2}\right) / 2=-0.02$.

Notice how the investor's buy LO quote for Asset 1 (as depicted in Figure 8(a)) varies as the process $S_{t}^{(1)}-S_{t}^{(2)}$ varies over time in Figure $7(\mathrm{~d})$. The discrete jumps in $\delta_{1, t}^{-}$are due to the state variable $s_{1}-s_{2}$ moving to another (discrete) grid point (recall that $g(t, \mathbf{s}, \mathbf{q}$ ) was solved numerically on a sparse grid for the spatial variable $\mathbf{s})$. In this plot, the cheaper Asset 1 is relative to Asset 2 (i.e. when $S_{t}^{(1)}-S_{t}^{(2)}$ is close to -0.02 ), the more willing the investor is to acquire an additional unit of Asset 1. Hence, she posts her limit buy order closer to the best bid for Asset 1 and her limit sell order closer to the best offer for Asset 2. Along this sample path, the investor manages to acquire a long position in Asset 1, as shown in 8(b), and is using her short position in Asset 2 to partially hedge away the risk associated with being long the underlying equity. 


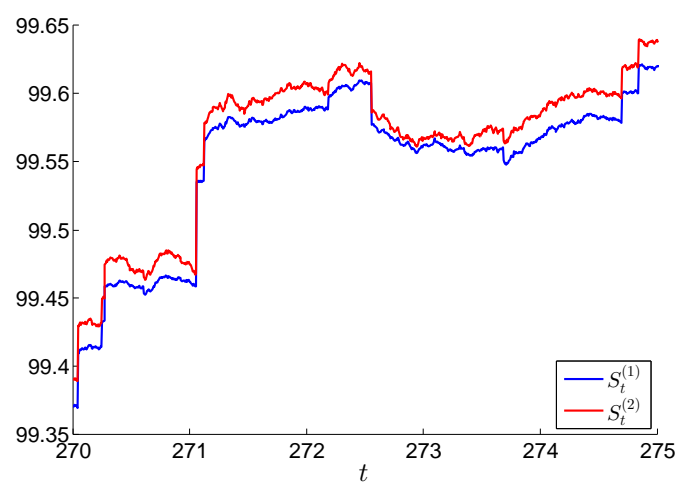

(a) Midprice Process

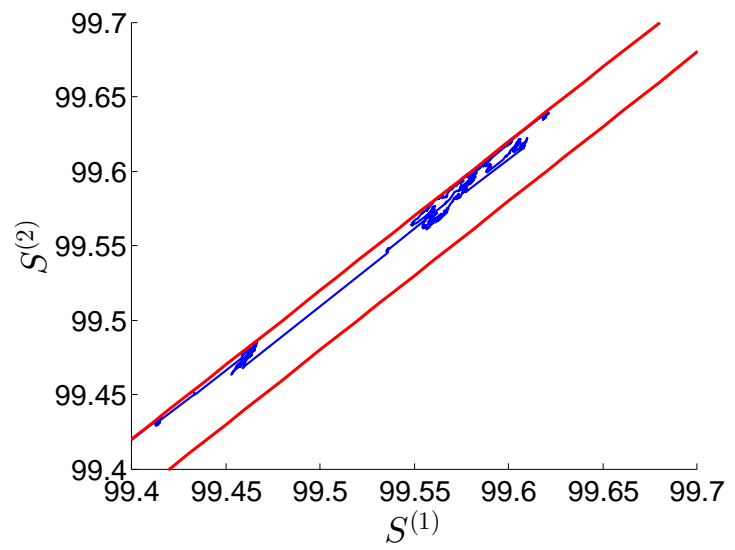

(c) Midprice Process

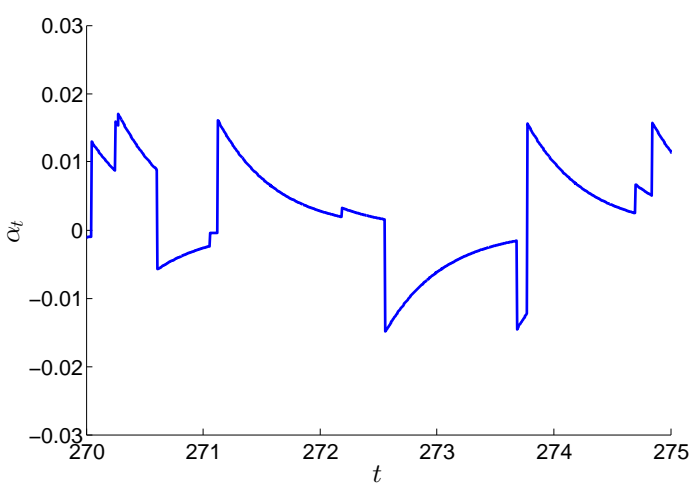

(b) Drift Process, $\alpha_{t}=\alpha_{t}^{1}=\alpha_{t}^{2}$

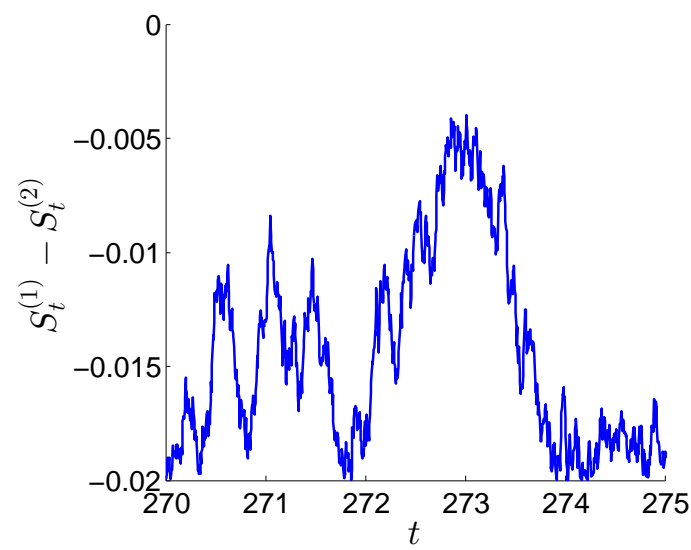

(d) Dimensionally Reduced Midprice Process

Figure 7: Sample paths of the mid-price process $\mathbf{S}_{t}$ and drift process $\boldsymbol{\alpha}_{t}$. The red lines in Figure 7(c) represent the boundary of the no-arbitrage region.

Although these adverse selection effects are present in the DGP, the trader cannot estimate the correct drift of the asset so she assumes that it is zero. Recall that the investor assumes that the dynamics of $\mathbf{S}_{t}$ are given by Assumption 1, and that this was the assumption used when solving the stochastic control problem above.

\subsection{Results of the Simulation Study}

Here we show how the investor's strategy performs when we vary the running inventory penalty $\phi$ and her exposure to adverse selection by varying the scale parameter $\epsilon$. The investor starts with zero inventory in both assets and will trade for $T=300$ seconds. We run 2,500 simulations and record the PnL for each run. Figure 9 provides histograms depicting the distribution of the terminal PnL for two levels of running inventory penalty. In Figure 9(a) we assume that the investor is not exposed to adverse selection costs by assuming $\epsilon=0$ and in Figure 9(b) we 


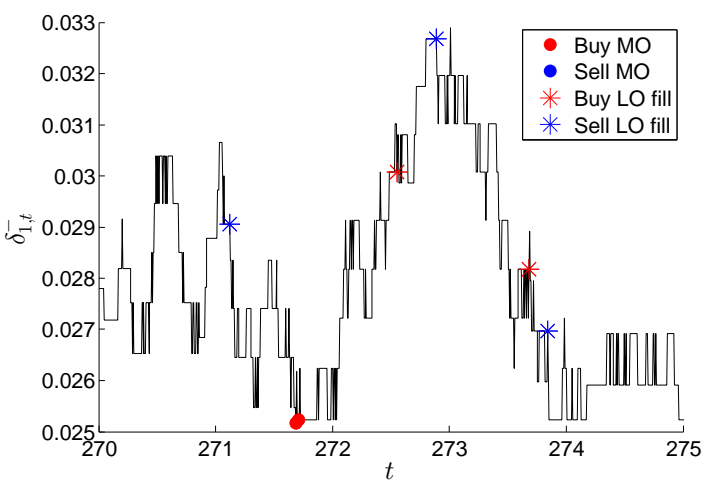

(a) Optimal Asset 1 Buy LO Depth

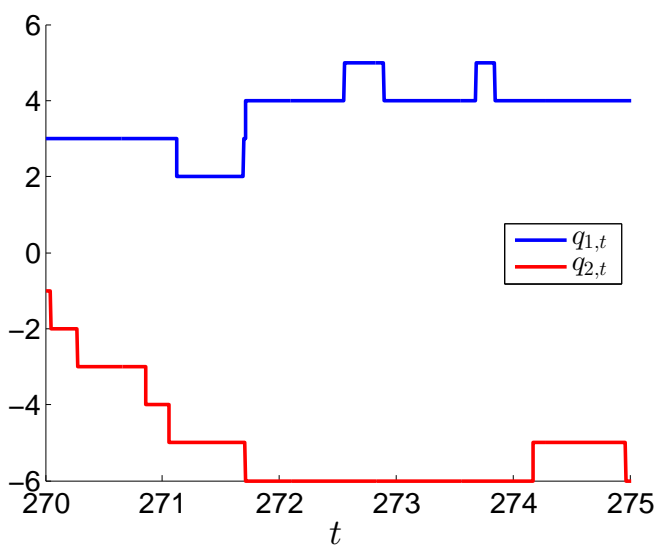

(b) Inventory Process

Figure 8: Sample paths of the investor's LO depth and her inventory level for $\phi=10$.

show the histogram of the PnLs when the investor is exposed to adverse selection effects by setting $\epsilon=0.02$.

However, when we increase the scale parameter $\epsilon$ in the jump size of the short-term drift process, we observe that the less inventory averse investor does not necessarily outperform her more inventory averse counterpart. This is because the less inventory averse investor is willing to hold larger inventories for a longer period of time which further exposes her to the drift of $\mathbf{S}_{t}$, while the more inventory averse investor cuts her losses early in an attempt to reduce inventory risk. The $\epsilon=0.04$ case in Figure 10 illustrates this result very well.

There is some debate about which risk metric better characterizes the riskiness of a strategy. Classical work in finance is typically only concerned with the terminal distribution of PnL (at time $T$ ); namely, its variance (or standard deviation) and possibly higher moments that give information about the weight of the tails. However, considering the penalty term in (11), we see that we are concerned with the optimal portfolio's risk throughout the entire time interval $[0, T]$, not just at the end. To this end, we classify a strategy's riskiness via the penalty $\int_{0}^{T} \ell\left(\mathbf{q}_{u}\right) d u$ and consider the mean of this quantity. This is what is termed mean inventory risk, see Cartea and Jaimungal (2015b) in the context of algorithmic trading.

Figures 10 and 11 provide 'efficient frontiers' comparing expected PnL against both standard deviation of PnL and mean inventory risk for a variety of adverse selection parameters ( $\epsilon$ and $\rho$, respectively). Decreasing $\phi$ (i.e. increasing inventory-risk appetite) corresponds to moving in the rightward direction of this plot. As stated in Table 2, $\phi$ ranges from 0.3 to 200.

Recall that the investor assumes that the drift of $\mathbf{S}_{t}$ is zero, when in fact there is a short-term component in the drift that affects mid-prices when informed traders send MOs. As outlined 


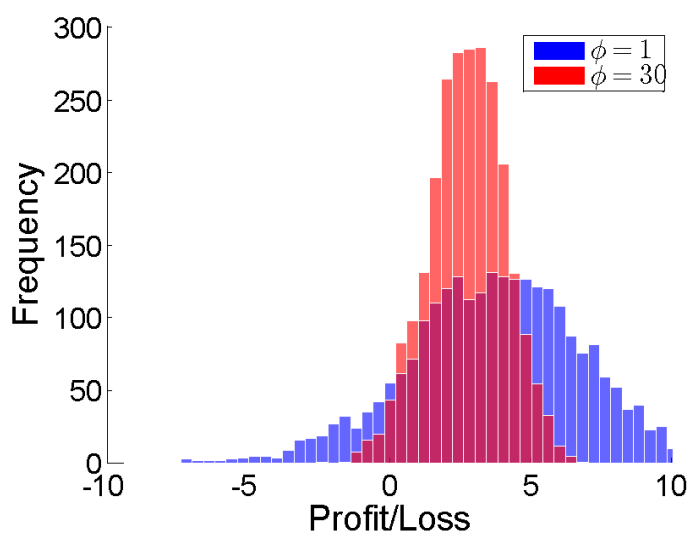

(a) $\epsilon=0$

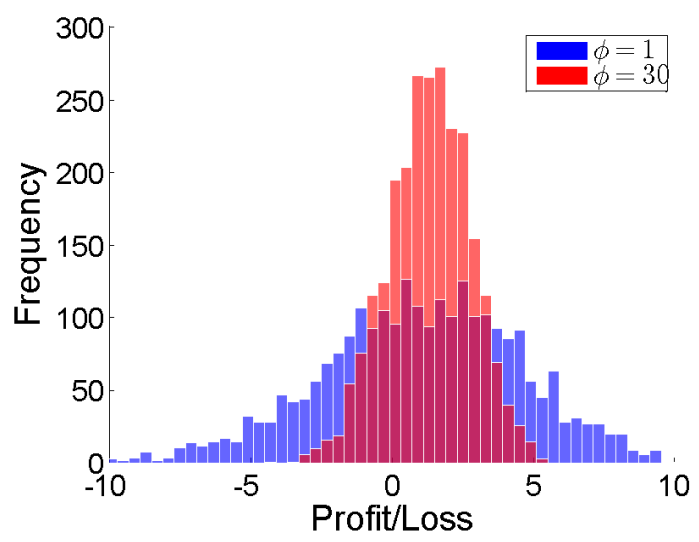

(b) $\epsilon=0.02$

Figure 9: Distribution of terminal profit and loss. The probability of an informed MO is fixed at $\rho=0.50$.

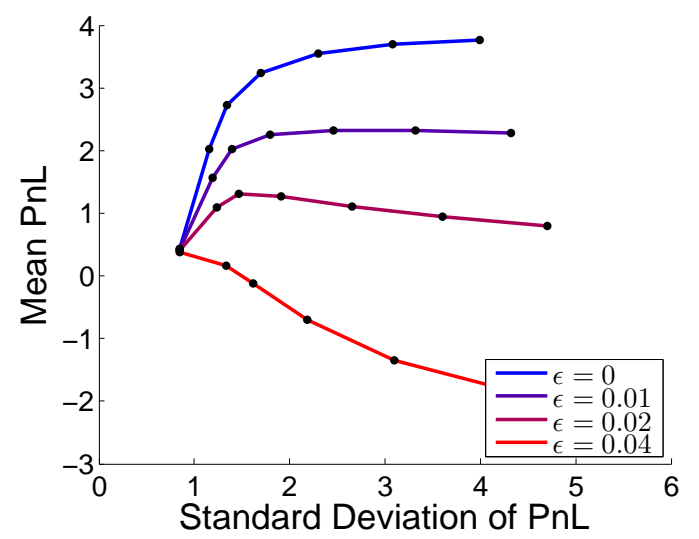

(a) Mean PnL vs Std Dev PnL

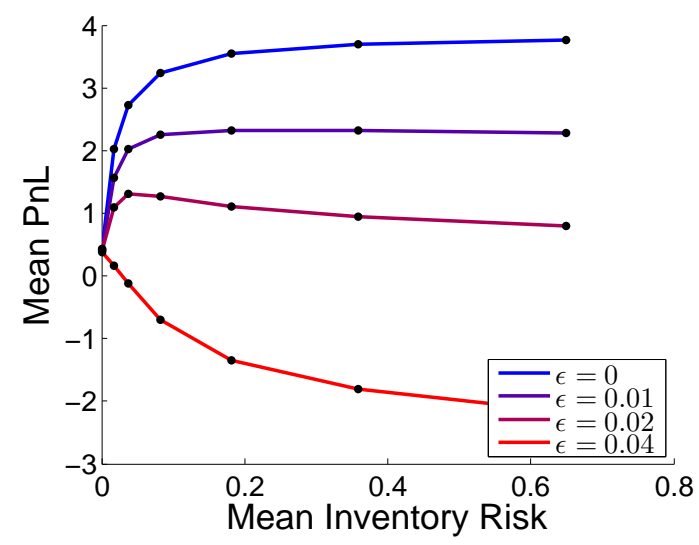

(b) Mean PnL vs Mean Inventory Risk

Figure 10: Performance of strategy for various $\phi$ with different drift impact scale parameters $\epsilon$. The probability of an informed MO is fixed at $\rho=0.50$.

in detail in Section 5.3, we have a scale parameter $\epsilon \geq 0$ that represents the magnitude of such effects. The larger the parameter, the stronger the effects of adverse selection are in the market. It is clear from Figure 10, that adverse selection generally has a negative effect on the investor's PnL. In the extreme case of $\epsilon=0.04$, these effects are so strong that decreasing the investor's inventory aversion actually decreases return because she is allowing her portfolio to be exposed to these effects for a longer period of time.

Up until this point, we have assumed that 50\% of all incoming MOs are from informed traders and thus have an impact on the drift of the mid-price. Recall that MOs of this type have two effects: an immediate effect and a persistent effect. If we increase the probability $\rho$ that 


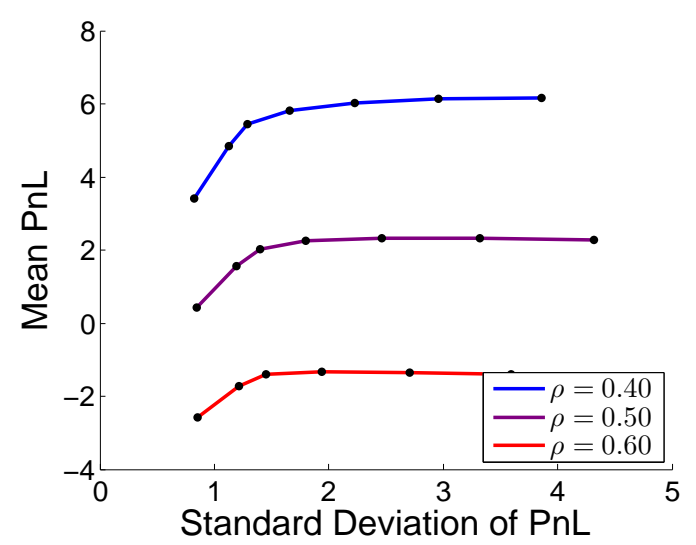

(a) Mean PnL vs Std Dev PnL

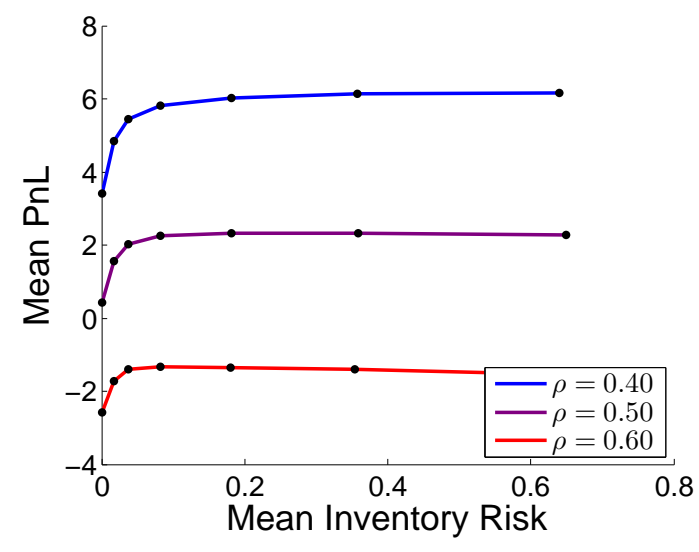

(b) Mean PnL vs Mean Inventory Risk

Figure 11: Performance of strategy for various $\phi$ with different probabilities of informed MOs $\rho$. The drift impact scale parameter is fixed at $\epsilon=0.01$.

an $\mathrm{MO}$ comes from an informed trader, then this will directly translate into an increase in the number of MOs that immediately move the market. Because this movement is always against the investor when her $\mathrm{LO}$ is filled, it will always have a negative impact on her $\mathrm{PnL}$ (in contrast to increasing $\epsilon$ where this was not the case). The resulting efficient frontiers are reported in Figure 11 for three differing values of $\rho$.

Figure 12 shows that the larger the inventory penalty parameter $\phi$ is, the more MOs are executed by the investor. Larger $\phi$ means that the investor imposes a higher penalty on running inventory, which induces higher mean reversion in inventories. This is attained by adjusting the depth of LOs and by executing MOs to unwind positions. For example, if the inventory is long, the sell LO will be posted very close to the mid-price, the buy LO far away from the mid-price, and if necessary execute a sell MO. One can also see that see that the larger $\phi$ is, the smaller the continuation region, so the boundary gets breached more often inducing MOs that force the investor to cross the bid-ask spread to bring $\mathbf{q}$ back into the continuation region.

Although there is symmetry between the buy and sell sides of the LOB, there is an intuitive explanation for the drastic difference in number of executed MOs between the two assets. Asset 2 has a higher total volatility, which increases the penalty for holding a position in it, hence, the continuation region is smaller in the $q_{2}$ direction than in the $q_{1}$ direction (as seen in Figure 2). 


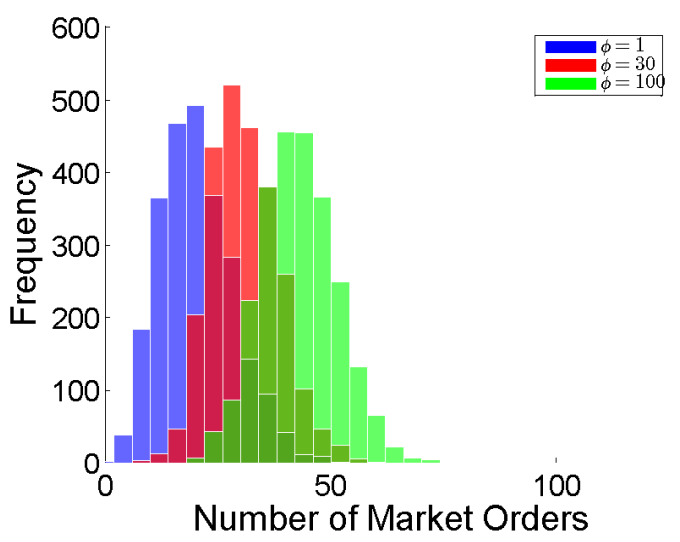

(a) Asset 1 - Market Buy

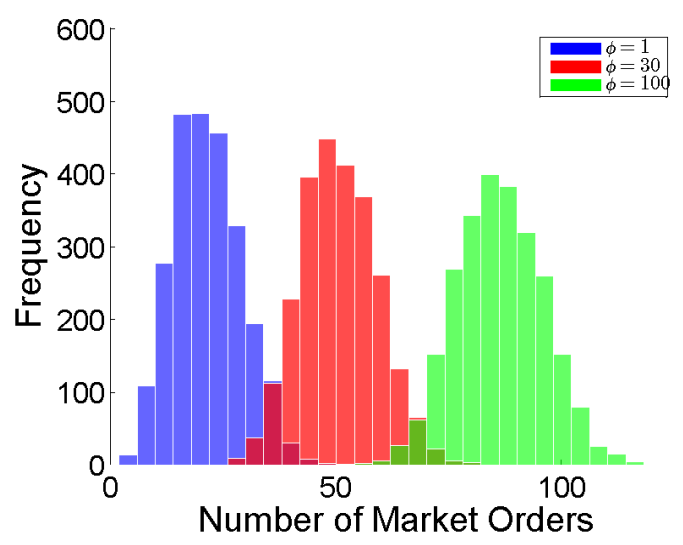

(c) Asset 2 - Market Buy

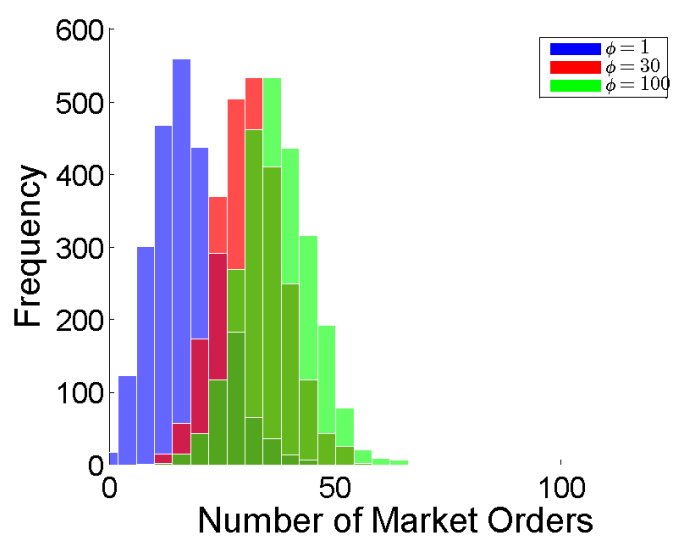

(b) Asset 1 - Market Sell

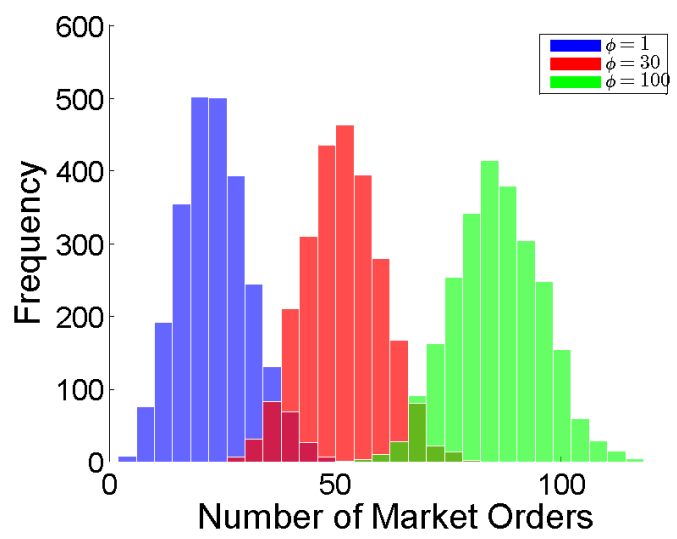

(d) Asset 2 - Market Sell

Figure 12: Distribution of the number of executed MOs for various inventory penalty levels when $\epsilon=0.01$ and $\rho=0.50$.

\section{Conclusions}

We developed a trading strategy which employs limit and market orders in a multi-asset economy where assets exhibit structural dependence. For example, i) trading in a futures contract and its underlying, or ii) trading in the first and second nearby futures and the 1-2 calendar spread. When structurally dependent assets trade with positive bid-ask spread, midprices live in a no-arbitrage region and we showed how an investor can take positions in these assets to maximize expected cash over a trading horizon.

We assumed that the investor's performance criteria is linear in wealth and penalizes inventory positions. Key to the strategy is the behaviour of mid-prices close to the edges of the no-arbitrage region. Price pressure from arbitrageurs and other traders push prices to the interior of the region, and in our framework this is modeled assuming that mid-prices follow 
a multivariate Brownian motion that is reflected on the boundaries of this region.

The optimal strategy developed here exhibits two dominant features which depend on how far the vector of mid-prices is from the no-arbitrage bounds. When mid-prices are sufficiently far from the no-arbitrage edges, the strategy behaves as that of a market maker who posts buy and sell LOs. However, these LOs are strategically placed in the book to not only control for inventory exposure, but also in anticipation of mid-prices approaching the edges of the no-arbitrage region where the strategy employs a mix of market and limit orders to execute statistical arbitrages.

We employed simulations to show the qualitative features of the optimal trading strategy. we observed that market orders, employed for statistical arbitrages and inventory management, play a key role in the success of the strategy. Limit orders are posted in the book to earn the spread, and to manage inventories where the instantaneous correlation between multiple assets is also used to partially hedge away the inventory risk associated with a large open position in the less liquid assets.

We provided an explicit numerical scheme to solve the derived HJBQVI and convergence results were discussed to ensure that the given discretization scheme converges to the true viscosity solution. Lastly, we derived a dimensional reduction in the spatial variable for the mid-price which significantly reduces the computational time of the numerical solution, thus making it viable for practical applications.

As electronic markets become more integrated as a result of trading strategies that exploit relationships in limit order books, the approach presented here will provide a framework to develop trading strategies that benefit from co-movements of financial instruments across asset classes. Machine learning and other data mining techniques could also be employed to model co-movements of assets and incorporate this information in the control problem developed above.

\section{References}

Almgren, R. (2003). Optimal execution with nonlinear impact functions and trading-enhanced risk. Applied Mathematical Finance 10(1), 1-18.

Almgren, R. and N. Chriss (2000). Optimal execution of portfolio transactions. Journal of Risk 3.

Avellaneda, M. and S. Stoikov. High-frequency trading in a limit order book. Quantitative Finance 8.

Barles, G. and P. Souganidis (1991). Convergence of approximation schemes for fully nonlinear second order equations. Asymptotic Analysis 4, 271-283. 
Bayraktar, E. and M. Ludkovski (2014). Liquidation in limit order books with controlled intensity. Mathematical Finance 24(4), 627-650.

Cartea, Á. and S. Jaimungal (2015a). Optimal execution with limit and market orders. Quantitative Finance 15(8), 1279-1291.

Cartea, Á. and S. Jaimungal (2015b). Risk metrics and fine tuning of high-frequency trading strategies. Mathematical Finance 25(3), 576-611.

Cartea, Á. and S. Jaimungal (2016). Algorithmic trading of co-integrated assets. International Journal of Theoretical and Applied Finance 19(06), 1650038.

Cartea, Á., S. Jaimungal, and R. Donnelly (2013). Algorithmic trading with model uncertainty. SSRN: http://ssrn.com/abstract=2310645.

Cartea, Á., S. Jaimungal, and J. Penalva (2015). Algorithmic and High-Frequency Trading. Cambridge University Press.

Cartea, Á., S. Jaimungal, and J. Ricci (2014). Buy low, sell high: A high-frequency trading perspective. SIAM Journal of Financial Mathematics 5, 415-444.

Forsyth, P., S. Kennedy, J. Tse, and H. Windcliff (2012). Optimal trade execution: a meanquadratic-variation approach. Journal of Economic Dynamics and Control 36, 1971-1991.

Guéant, O., C.-A. Lehalle, and J. Fernandez Tapia (2012). Optimal portfolio liquidation with limit orders. SIAM Journal on Financial Mathematics 3(1), 740-764.

Guéant, O., C.-A. Lehalle, and J. Fernandez Tapia (2013). Dealing with the inventory risk: a solution to the market making problem. Mathematics and Financial Economics 7(4), $477-507$.

Guilbaud, F. and H. Pham (2013). Optimal high frequency trading with limit and market orders. Quantitative Finance 13(1), 79-94.

Guilbaud, F. and H. Pham (2015). Optimal high-frequency trading in a pro rata microstructure with predictive information. Mathematical Finance 25(3), 545-575.

Ho, T. and H. R. Stoll (1981). Optimal dealer pricing under transactions and return uncertainty. Journal of Financial Economics 9, 47-73.

Kharroubi, I. and H. Pham (2010). Optimal portfolio liquidation with execution cost and risk. SIAM Journal on Financial Mathematics 1, 897-931.

Leung, T. and X. Li (2015). Optimal mean reversion trading with transaction costs and stop-loss exit. International Journal of Theoretical and Applied Finance, 1550020.

Øksendal, B. and A. Sulem (2007). Applied Stochastic Control of Jump Diffusions, Volume 2. Berlin, Heidelberg, New York: Universitext, Springer Verlag. 
Tourin, A. and P. Lintilhac (2017). Model-based pairs trading in the bitcoin markets. Quantitative Finance 17(5), 703-716.

Tourin, A. and R. Yan (2013). Dynamic pairs trading using the stochastic control approach. Journal of Economic Dynamics and Control 37(10), 1972 - 1981.

\section{Appendix A. Evaluating Non-Trivial Terms in the Numerical Scheme of Section 4.2}

This section illustrates how to apply the dimensional reduction to terms where the result is non-trivial. All expressions are given explicitly for the $n=2$ example. In what follows, all functions are evaluated at a fixed inventory level, $\mathbf{q}$, and this dependence is suppressed.

Appendix A.1. The Mixed Partial Term

For $i \neq j$, we numerically approximate the following term that appears in (22):

$$
\frac{\partial^{2} g}{\partial s_{i} \partial s_{j}} \approx \frac{g\left(s_{i}+d \tilde{s}, s_{j}+d \tilde{s}, \cdot\right)-g\left(s_{i}+d \tilde{s}, \cdot\right)-g\left(s_{j}+d \tilde{s}, \cdot\right)+g(\cdot)}{d \tilde{s}^{2}},
$$

where $d \tilde{s}$ is the step size of the finite difference approximation. Note that if we require the grids to line up in our $n=2$ example, we must have $d \tilde{s}=d s$, where $d s$ is the step size on the dimensionally reduced grid given in Appendix A.3.

\section{Appendix A.2. Dimensional Reduction}

Recall that Corollary 10 states that there exists $v \in \mathbb{R}^{n} \backslash\{0\}$ such that $D_{v} g=0$. In our example, $v=\mathbf{1}$, so we take $\mathbf{s}:=s_{1}-s_{2}$.

Continuing from (A.1), we then have

$$
\frac{\partial^{2} g}{\partial s_{1} \partial s_{2}} \approx \frac{2 g(\cdot)-g(\mathbf{s}-d s, \cdot)-g(\mathbf{s}+d s, \cdot)}{d s^{2}}
$$

where the right hand side is defined on the dimensionally reduced grid and taken at the previous time step $(t+d t)$.

For the two-dimensional illustrative example studied in this paper, the full numerical approximation for the diffusive term in $\mathcal{L} g$ is then given by

$$
\frac{g(\mathbf{s}+d s, t+d t)+g(\mathbf{s}-d s, t+d t)-2 g(\mathbf{s}, t+d t)}{2 d s^{2}}\left(\left[\boldsymbol{\sigma} \boldsymbol{\sigma}^{\prime}\right]_{11}+\left[\boldsymbol{\sigma} \boldsymbol{\sigma}^{\prime}\right]_{22}-2\left[\boldsymbol{\sigma} \boldsymbol{\sigma}^{\prime}\right]_{12}\right) .
$$


For $\mathbf{s} \in \partial \mathcal{A}$, numerically impose $(\mathbf{q}+\nabla g) \cdot \boldsymbol{n}=0$. Take $d \bar{s}=c_{0} d s$ so the grids match up, where $c_{0}$ depends only on the choice of orthogonal basis of $B$.

In our $n=2$ example, we have the normal vector $\boldsymbol{n}=(1,-1)$ and $c_{0}=1$ resulting in $\nabla g \approx \mp\left(\frac{g(\mathbf{s})-g(\mathbf{s} \pm d s)}{d s}, \frac{g(\mathbf{s} \pm d s)-g(\mathbf{s})}{d s}\right)$ with the upper (lower) symbol for reflection on the upper (lower) boundary when viewed graphically. The relation is only approximate because we are using a finite difference method. Finally, for $\mathbf{s} \in \partial \mathcal{A}$, we have

$$
g(\mathbf{s}) \approx g(\mathbf{s} \pm d s) \pm \frac{\left(q_{1}-q_{2}\right) d s}{2} .
$$

Appendix A.4. Explicit Example when $n=3$

The analogue of the right-most coefficient in (A.3) is given by

$$
\left[\boldsymbol{\sigma} \boldsymbol{\sigma}^{\prime}\right]_{11}+\left[\boldsymbol{\sigma} \boldsymbol{\sigma}^{\prime}\right]_{22}+\left[\boldsymbol{\sigma} \boldsymbol{\sigma}^{\prime}\right]_{33}-2\left[\boldsymbol{\sigma} \boldsymbol{\sigma}^{\prime}\right]_{12}-2\left[\boldsymbol{\sigma} \boldsymbol{\sigma}^{\prime}\right]_{13}+2\left[\boldsymbol{\sigma} \boldsymbol{\sigma}^{\prime}\right]_{23}
$$

and, for $\mathbf{s} \in \partial \mathcal{A}$, we have

$$
g(\mathbf{s}) \approx g(\mathbf{s} \pm d s) \pm \frac{\left(q_{1}-q_{2}-q_{3}\right) d s}{3}
$$

